Research Article

\title{
Reachable Set Estimation for Uncertain Markovian Jump Systems with Time-Varying Delay and Disturbances
}

\author{
Shouwei Zhou, Jiangliu Gu, Changchun Shen (D), and Min Jiang \\ School of Data Science and Information Engineering, Guizhou Minzu University, Guiyang, China \\ Correspondence should be addressed to Changchun Shen; sccyjs2008@163.com
}

Received 4 November 2020; Revised 27 December 2020; Accepted 4 January 2021; Published 8 February 2021

Academic Editor: Efthymios G. Tsionas

Copyright $(2021$ Shouwei Zhou et al. This is an open access article distributed under the Creative Commons Attribution License, which permits unrestricted use, distribution, and reproduction in any medium, provided the original work is properly cited.

\begin{abstract}
In this paper, we are concerned with the problem of reachable set estimation for uncertain Markovian jump systems with timevarying delays and disturbances. The main consideration is to find a proper method to obtain the no-ellipsoidal bound of the reachable set of Markovian jump system as small as possible. Based on an augmented Lyapunov-Krasovskii functional, by dividing the time-varying delay into two nonuniform subintervals, more general delay-dependent stability criteria for the existence of a desired ellipsoid are derived. An optimized integral inequality which is based on distinguished Wirtinger integral inequality and reciprocally convex combination inequality is used to deal with the integral terms. Finally, numerical examples are presented to demonstrate the effectiveness of the theoretical results.
\end{abstract}

\section{Introduction}

The reachable set for a dynamic system with disturbances is a set that bounds the state trajectories starting from the origin by inputs with peak value. In practice and engineering applications, many dynamical systems may cause abrupt variations in their structure due to stochastic failures or repairs of the components, changes in the interconnections of subsystems, sudden environment changes, and so on. Markovian jump systems, modeled by a set of subsystems with transitions among the models determined by a Markov chain taking values in a finite set, have appealed to a lot of researchers in the control community. In the past few decades, the Markovian jump systems have been extensively studied (see [1-3] and the references therein).

For the bound of reachable sets for linear systems without any time delay, we can find a well-known result which has been formulated in terms of linear matrix inequality (LMI) [4], and it is widely used to design control systems that have saturating actuators $[5,6]$. However, timedelay phenomenon is frequently encountered in many practical systems, such as biological systems, chemical systems, hydraulic systems, and electrical networks. In recent years, the problem of reachable set estimation for time- delay systems has attracted much attention. Then, an increasing number of researchers have devoted their efforts to the problem of reachable set estimation [7-11]. In [7], a delay-dependent condition for an ellipsoid bounding the set of reachable states was presented by using the Lyapunov-Razumikhin function and the $S$-procedure. Five nonconvex scalar parameters have to be treated as tuning parameters to find the smallest possible ellipsoid. Based on a relaxed Lyapunov-Krasovskii function, the delay-dependent and delay-rate-dependent conditions for the existence of a desired ellipsoid are obtained [8]. Chen and Zhong [10] studied the reachable set of neutral systems with perturbations and uncertainties via novel inequality. In [11], the time-varying delay is split into two nonuniform subintervals based on the Lyapunov-Krasovskii functional, and using the well-known Wirtinger integral inequality and reciprocating convex combination inequality, the RSE boundary of the time-delay system is obtained.

However, the Markov jump system is different from the general time-delay system. It is a random system with multiple modes. The jump transfer between the various modes of the system is determined by a set of Markov chains. In practical application, the equation of state of the system often has some randomness. On the one hand, the stability 
and stability of Markov jump system have been widely studied [12-16]. On the other hand, there are few studies on the reachable set estimation problem of the Markov jump system (see [17-20] and the references therein). Therefore, this paper studies the reachable set estimation of uncertain Markov jump system.

Besides, in real life, parameter uncertainty is inevitable in the mathematical modeling due to the failure or maintenance of parts, external perturbations, parameter fluctuations, data errors and the change of connection mode of subsystems, etc. Therefore, inspired by the issues discussed above, the problem of reachable set estimation for uncertain Markov jump systems with time-varying delays and disturbance is investigated in this study. The interval is divided by time-varying delay split based on Lyapunov-Krasovskii functional, and the partial integral term of derivative of Lyapunov function is optimized by using Wirtinger-based inequality and reciprocating convex matrix inequality.
Finally, numerical examples are given to illustrate the validity of the results.

Notations: the notations used throughout the paper are fairly standard. The superscript " $T$ " stands for matrix transposition; $\mathfrak{R}^{n}$ denotes the $n$-dimensional Euclidean space; the notation $P>0$ means that $P$ is a positive definite matrix; $I_{n}$ and $0^{n \times n}$ represent identity matrix and zero matrix with dimension $n$, respectively; and sym $\left(X_{11}\right)=X_{11}+X_{11}^{T}$. In symmetric block matrices, we use an asterisk (*) to represent a term which is induced by symmetry. Matrices, if their dimensions are not explicitly stated, are assumed to be compatible for algebraic operations.

\section{Problem Statement}

Consider the following Markov jump systems with uncertainties:

$$
\left\{\begin{array}{l}
\dot{x}(t)=\left(A_{\left(t, r_{t}\right)}+\Delta A_{\left(t, r_{t}\right)}(t)\right) x(t)+\left(B_{\left(t, r_{t}\right)}+\Delta B_{\left(t, r_{t}\right)}(t)\right) x(t-h(t))+\left(D_{\left(t, r_{t}\right)}+\Delta D_{\left(t, r_{t}\right)}(t)\right) \omega(t) \\
x(t) \equiv 0, \quad \forall t \in\left[-h_{2}, 0\right]
\end{array}\right.
$$

where $x(t) \in \mathfrak{R}^{n}$ is the state vector and $\omega(t) \in \mathfrak{R}^{m}$ is the disturbance which satisfies

$$
\omega^{T}(t) \omega(t) \leq \omega_{m}^{2} \leq 1
$$

The discrete time-varying delay $h(t)>0$ satisfies

$$
0 \leq h_{1} \leq h(t) \leq h_{2}
$$

where $h_{1}, h_{2}$ are constants and $h_{m}=\gamma h_{1}+(1-\gamma) h_{2}$, $0 \leq \gamma \leq 1$. $\left\{r_{t}, t \geq 0\right\}$ is a Markovian process taking values on the probability space in a finite state $\wp=\{1,2, \ldots, N\}$ with generator $\Lambda=\left\{\lambda_{i j}\right\},(i, j \in \wp)$ given by

$$
\operatorname{Pr}\left\{r_{t+\Delta}=j \mid r_{t}=i\right\}= \begin{cases}\lambda_{i j} \Delta+o(\Delta), & j \neq i, \\ 1+\lambda_{i j} \Delta+o(\Delta), & j=i,\end{cases}
$$

where $\Delta>0, \lim _{\Delta \longrightarrow 0^{+}}(o(\Delta)) / \Delta=0, \lambda_{i j} \geq 0$, for $j \neq i$ is the transition probability from mode $i$ at time $t$ to mode $j$ at time $t+\Delta, \lambda_{i i}=-\sum_{j=1, j \neq i}^{N} \lambda_{i j} . A_{\left(t, r_{t}\right)}, B_{\left(t, r_{t}\right)}$, and $D_{\left(t, r_{t}\right)}$ are known constant matrices of the Markovian process. For notational simplicity, when $\left(t, r_{t}\right)=i, i \in \wp$, the matrix $A_{\left(t, r_{t}\right)}$ will be represented by $A_{i}$, and the other symbols are similarly defined.

Since the state transition probability of the Markovian jump process considered in this paper is partially known, the transition probability matrix of Markovian jumping process $\Lambda$ is defined as

$$
\Lambda=\left(\begin{array}{cccc}
\lambda_{11} & ? & \cdots & \lambda_{1 N} \\
? & \lambda_{22} & \cdots & \lambda_{2 N} \\
\vdots & \vdots & \ddots & \vdots \\
\lambda_{N 1} & ? & \cdots & \lambda_{N N}
\end{array}\right)
$$

where? represents the unknown transition rate. For notational clarity, $\forall i \in \wp$, and the set $U^{i}=U_{k}^{i} \cup U_{u k}^{i}$ with

$$
\begin{aligned}
U_{k}^{i} & \triangleq\left\{j: \lambda_{i j} \text { is known for } j \in \wp\right\}, \\
U_{u k}^{i} & \triangleq\left\{j: \lambda_{i j} \text { is unknown for } j \in \wp\right\} .
\end{aligned}
$$

Moreover, if $U_{k}^{i} \neq \varnothing$, it is further described as $U_{k}^{i}=\left\{k_{1}^{i}, k_{2}^{i}, \ldots, k_{m}^{i}\right\}$, where $m$ is a non-negation integer with $1 \leq m \leq N$ and $k_{j}^{i} \in Z^{+}, \quad 1 \leq k_{j}^{i} \leq N, j=1,2, \ldots, m$ represent the known element of the $i$ th row and $j$ th column in the state transition probability matrix $\Lambda$.

Besides, $\Delta A_{i}, \Delta B_{i}$, and $\Delta D_{i}$ are the parametric uncertainties in system (1), which are assumed to be in the following form:

$$
\left[\Delta A_{i}, \Delta B_{i}, \Delta D_{i}\right]=L_{i} K_{i}(t)\left[E_{i a}, E_{i b}, E_{i d}\right]
$$

where $K_{i}(t)$ is an unknown real and possibly time-varying matrix with Lebesgue measurable elements satisfying

$$
K_{i}^{T}(t) K_{i}(t) \leq I
$$

and $L_{i}, E_{i a}, E_{i b}$, and $E_{i d}$ are known real constant matrices which characterize how the uncertainty enters the nominal matrices $A_{i}, B_{i}$, and $D_{i}$.

Before proceeding further, system (1) can be written as

$$
\left\{\begin{array}{l}
\dot{x}(t)=A_{i} x(t)+B_{i} x(t-h(t))+D_{i} \omega(t)+L_{i} u, \\
z=E_{i a} x(t)+E_{i b} x(t-h(t))+E_{i d} \omega(t),
\end{array}\right.
$$

with the constraint: $u=K_{i}(t) z$.

We further have 


$$
\begin{aligned}
u^{T} u \leq & {\left[E_{i a} x(t)+E_{i b} x(t-h(t))+E_{i d} \omega(t)\right]^{T} } \\
\cdot & {\left[E_{i a} x(t)+E_{i b} x(t-h(t))+E_{i d} \omega(t)\right] . }
\end{aligned}
$$

For the sake of brevity, $x(t)$ is used to represent the solution of the system under initial conditions $x\left(t, t_{0}, x_{0}\right)$, and $\{x(t), t\}$ satisfies the initial condition $\left\{x(0), r_{0}\right\}$. And its weak infinitesimal generator, acting on function $V$, is defined in [21].

$$
\begin{aligned}
L V(x(t), t, i)= & \lim _{\Delta \longrightarrow 0^{+}} \frac{1}{\Delta}\left[\varepsilon \left(V\left(x(t+\Delta), t+\Delta, r_{t+\Delta}\right)\right.\right. \\
& \left.\left.\mid x(t), r_{t}=i\right)-V(x(t), t, i)\right] .
\end{aligned}
$$

A reachable set that bounds the state of system (1) is defined by

$$
\mathfrak{R}_{x}=\left\{x(t) \in \mathfrak{R}^{n} \mid x(t), \omega(t) \text { satisfy (1) and (2) }\right\} .
$$

Based on the ideas proposed in [4], this reachable set estimation problem can be transformed into the problem of finding an ellipsoid to bound the $\mathfrak{R}_{x}$. We will bound $\mathfrak{R}_{x}$ by an ellipsoid of the form

$$
\mathfrak{J}(P) \triangleq\left\{\xi(t) \in \mathfrak{R}^{n}: \xi^{T}(t) P \xi(t) \leq 1 ; \quad P>0\right\} .
$$

Before proceeding further, we will state well-known lemmas.

Lemma 1 (see [4]). Let $V(t, x(0))=0$ and $\omega^{T}(t) \omega(t) \leq \omega_{m}^{2}$; if

$$
L V\left(t, x_{t}\right)+\alpha V\left(t, x_{t}\right)-\beta \omega^{T}(t) \omega(t) \leq 0, \quad \alpha>0, \beta>0,
$$

then we have $V\left(t, x_{t}\right) \leq(\beta / \alpha) \omega_{m}^{2}$ for $\forall t \geq 0$.

Lemma 2 (see [22]). For any positive definite matrix $M \in \mathbb{R}^{n \times n}$, scalar $h_{2}>h_{1} \geq 0$, and vector function $\omega:\left[h_{1}, h_{2}\right] \mapsto \mathfrak{R}^{n}$ such that the integrations concerned are well defined,

$$
\begin{aligned}
& -\left(h_{2}-h_{1}\right)\left(\int_{t-h_{2}}^{t-h_{1}} \omega^{T}(s) M \omega(s) \mathrm{d} s\right) \\
& \leq-\left(\int_{t-h_{2}}^{t-h_{1}} \omega(s) \mathrm{d} s\right)^{T} M\left(\int_{t-h_{2}}^{t-h_{1}} \omega(s) \mathrm{d} s\right) .
\end{aligned}
$$

Lemma 3 (see $[23,24]$ ). For given a matrix $R>0$, the following inequality holds for all continuously differentiable functions $\omega(t)$ in $\omega:[a, b] \mapsto \mathbb{R}^{n}$ :

$$
\begin{aligned}
& \int_{a}^{b} \dot{\omega}^{T}(s) R \dot{\omega}(s) \mathrm{d} s \geq \frac{1}{b-a} \Omega_{1}^{T} R \Omega_{1}+\frac{3}{b-a} \Omega_{2}^{T} R \Omega_{2} \\
& \quad+\frac{5}{b-a} \Omega_{3}^{T} R \Omega_{3},
\end{aligned}
$$

where

$$
\begin{aligned}
\Omega_{1}= & \omega(b)-\omega(a), \\
\Omega_{2}= & \omega(b)+\omega(a)-\frac{2}{b-a} \int_{a}^{b} \omega(s) \mathrm{d} s, \\
\Omega_{3}= & \omega(b)-\omega(a)+\frac{6}{b-a} \int_{a}^{b} \omega(s) \mathrm{d} s \\
& -\frac{12}{(b-a)^{2}} \int_{a}^{b} \int_{u}^{b} \omega(s) \mathrm{d} s \mathrm{~d} u .
\end{aligned}
$$

Lemma 4 (reciprocally convex combination inequality [25]). For all vectors $\xi \in \mathfrak{R}^{n}$, the function

$$
H(\alpha, Q)=\frac{1}{\alpha} \xi^{T} W_{1}^{T} Q W_{1} \xi+\frac{1}{1-\alpha} \xi^{T} W_{2}^{T} Q W_{2} \xi,
$$

where $\alpha \in(0,1), W_{1}, W_{2}$ and $Q$ are matrices with appropriate dimensions. Then, the following inequality holds:

$$
\min _{\alpha \in(0,1)} H(\alpha, Q) \geq \xi^{T}\left[\begin{array}{l}
W_{1} \\
W_{2}
\end{array}\right]^{T}\left[\begin{array}{ll}
Q & X \\
* & Q
\end{array}\right]\left[\begin{array}{l}
W_{1} \\
W_{2}
\end{array}\right] \xi
$$

if there exists a matrix $X$ such that $\left[\begin{array}{cc}Q & X \\ * & Q\end{array}\right]>0$.

Lemma 5 (Schur complement [26]). Given constant symmetric matrices $\sum_{1}, \sum_{2}, \sum_{3}$, where $\sum_{1}=\sum_{1}^{T}$ and $\sum_{2}=\sum_{2}^{T}>0$, then $\sum_{1}+\sum_{3}^{T} \sum_{2}^{-1} \sum_{3}<0$ holds if and only if

$$
\left[\begin{array}{cc}
\sum_{1} & \sum_{3}^{T} \\
\sum_{3} & -\sum_{2}
\end{array}\right]<0 \text { or }\left[\begin{array}{cc}
-\sum_{2} & \sum_{3} \\
\sum_{3}^{T} & \sum_{1}
\end{array}\right]<0 .
$$

\section{Main Results}

In this section, some delay-dependent criteria for the existence of ellipsoid $\mathfrak{\Im}(P)$ bounding the states of system (1) will be obtained. The notations for some matrices are defined in Appendixes.

Theorem 1. Consider the uncertain Markov jump system (1) with constraints (2) and (3); if there exist real matrices $P_{i}=\left[\begin{array}{ccccc}P_{i 11} & P_{i 12} & P_{i 13} & P_{i 14} & P_{i 15} \\ * & P_{i 22} & P_{i 23} & P_{i 24} & P_{i 25} \\ * & * & P_{i 33} & P_{i 34} & P_{i 35} \\ * & * & * & P_{i 44} & P_{i 45} \\ * & * & * & * & P_{i 55}\end{array}\right]>0, \quad W_{i}>0(i=1,2, \ldots$, N), $\widetilde{P}_{1, i}>0, Q_{1}>0, Q_{2}>0, Q_{3}>0$ and $R_{i}>0(i=1, \ldots, 6)$, $\widetilde{R}_{5}=\left[\begin{array}{ccc}R_{5} & 0 & 0 \\ 0 & 3 R_{5} & 0 \\ 0 & 0 & 5 R_{5}\end{array}\right], \quad \widetilde{R}_{6}=\left[\begin{array}{ccc}R_{6} & 0 & 0 \\ 0 & 3 R_{6} & 0 \\ 0 & 0 & 5 R_{6}\end{array}\right]$, any matrices $M_{i}(i=1,2), X=\left[\begin{array}{lll}X_{11} & X_{12} & X_{13} \\ X_{21} & X_{22} & X_{23} \\ X_{31} & X_{32} & X_{33}\end{array}\right], \quad Y=\left[\begin{array}{lll}Y_{11} & Y_{12} & Y_{13} \\ Y_{21} & Y_{22} & Y_{23} \\ Y_{31} & Y_{32} & Y_{33}\end{array}\right]$ 
with appropriate dimension such that $\left[\begin{array}{cc}\widetilde{R}_{5} & X \\ * & \widetilde{R}_{5}\end{array}\right]>0$, $\left[\begin{array}{cc}\widetilde{R}_{6} & Y \\ * & \widetilde{R}_{6}\end{array}\right]>0, P_{i} \geq\left[\begin{array}{ccccc}\widetilde{P}_{1, i} & 0 & 0 & 0 & 0 \\ * & 0 & 0 & 0 & 0 \\ * & * & 0 & 0 & 0 \\ * & * & * & 0 & 0 \\ * & * & * & * & 0\end{array}\right]$, scalar $\varepsilon>0$ satisfying the following matrix inequality:

$$
\begin{aligned}
& \Psi_{i}+\Phi_{1}<0, \\
& \widehat{\Psi}_{i}+\Phi_{2}<0, \\
& \widehat{\Psi}_{i}+\Phi_{3}<0, \\
& \Psi_{i}+\Pi_{1}<0, \\
& \widehat{\Psi}_{i}+\Pi_{2}<0, \\
& P_{j}-W_{i} \leq 0, \quad j \in U_{u k}^{i}, j \neq i,
\end{aligned}
$$

$$
P_{j}-W_{i} \geq 0, \quad j \in U_{u k}^{i}, j=i,
$$

where $\Psi_{i}, \widehat{\Psi}_{i}, \Phi_{1}, \Phi_{2}, \Phi_{3}, \Pi_{1}$, and $\Pi_{2}$, are defined in Appendix $A$. Then, the reachable sets of system (1) with (2) and (3) are bounded by boundaries $\cap_{i=1}^{N} \mathfrak{\Im}\left(\widetilde{P}_{1, i}\right)$, which is defined in (12).

Proof. We choose the following Lyapunov-Krasovskii functional candidate as follows:

$$
V\left(t, x_{t}\right)=\sum_{i=1}^{5} V_{i}\left(t, x_{t}\right)
$$

where

$$
V_{1}(t, x(t))=\eta^{T}(t)\left[\begin{array}{cccc}
P_{i 11} & P_{i 12} & P_{i 13} & P_{i 14} \\
* & P_{i 22} & P_{i 23} & P_{i 24} \\
* & * & P_{i 33} & P_{i 34} \\
* & * & * & P_{i 44}
\end{array}\right] \eta(t)
$$

with

$$
\begin{aligned}
\eta^{T}(t)= & \left\{x^{T}(t)\left(\int_{t-h_{1}}^{t} x(s) \mathrm{d} s\right)^{T}\left(\int_{t-h_{m}}^{t-h_{1}} x(s) \mathrm{d} s\right)^{T}\left(\int_{t-h_{2}}^{t-h_{m}} x(s) \mathrm{d} s\right)^{T}\right\}, \\
V_{2}\left(t, x_{t}\right)= & \int_{t-h_{1}}^{t} e^{\alpha(s-t)} x^{T}(s) Q_{1} x(s) \mathrm{d} s+\int_{t-h_{m}}^{t-h_{1}} e^{\alpha(s-t)} x^{T}(s) Q_{2} x(s) \mathrm{d} s+\int_{t-h_{2}}^{t-h_{m}} e^{\alpha(s-t)} x^{T}(s) Q_{3} x(s) \mathrm{d} s, \\
V_{3}\left(t, x_{t}\right)= & h_{1} \int_{-h_{1}}^{0} \int_{t+\theta}^{t} e^{\alpha(s-t)} x^{T}(s) R_{1} x(s) \mathrm{d} s \mathrm{~d} \theta \\
& +\left(h_{m}-h_{1}\right) \int_{-h_{m}}^{-h_{1}} \int_{t+\theta}^{t} e^{\alpha(s-t)} x^{T}(s) R_{2} x(s) \mathrm{d} s \mathrm{~d} \theta \\
& +\left(h_{2}-h_{m}\right) \int_{-h_{2}}^{-h_{m}} \int_{t+\theta}^{t} e^{\alpha(s-t)} x^{T}(s) R_{3} x(s) \mathrm{d} s \mathrm{~d} \theta \\
V_{4}\left(t, x_{t}\right)= & h_{1} \int_{-h_{1}}^{0} \int_{t+\theta}^{t} e^{\alpha(s-t)} \dot{x}^{T}(s) R_{4} \dot{x}(s) \mathrm{d} s \mathrm{~d} \theta \\
V_{5}\left(t, x_{t}\right)= & \left(h_{m}-h_{1}\right) \int_{-h_{m}}^{-h_{1}} \int_{t+\theta}^{t} e^{\alpha(s-t)} \dot{x}^{T}(s) R_{5} \dot{x}(s) \mathrm{d} s \mathrm{~d} \theta+\left(h_{2}-h_{m}\right) \int_{-h_{2}}^{-h_{m}} \int_{t+\theta}^{t} e^{\alpha(s-t)} \dot{x}^{T}(s) R_{6} \dot{x}(s) \mathrm{d} s \mathrm{~d} \theta \\
P_{i}= & {\left[P_{i, j}\right]_{5 \times 5}, } \\
h_{m}= & \gamma h_{1}+(1-\gamma) h_{2}, 0 \leq \gamma \leq 1 .
\end{aligned}
$$

Taking derivative of $V\left(t, x_{t}\right)$ along the trajectories of system (1), we can obtain the following:

$$
L V=L V_{1}+L V_{2}+L V_{3}+L V_{4}+L V_{5}
$$

where 


$$
\begin{aligned}
& L V_{1}\left(t, x_{t}\right)=2 \eta^{T}(t) P_{i} \dot{\eta}(t)+\eta^{T}(t)\left(\sum_{j=1}^{N} \lambda_{i j} P_{j}\right) \eta(t) \\
& =2\left[\begin{array}{ll}
\eta^{T}(t) & u^{T}
\end{array}\right]\left[\begin{array}{ccccc}
P_{i 11} & P_{i 12} & P_{i 13} & P_{i 14} & P_{i 15} \\
* & P_{i 22} & P_{i 23} & P_{i 24} & P_{i 25} \\
* & * & P_{i 33} & P_{i 34} & P_{i 35} \\
* & * & * & P_{i 44} & P_{i 45} \\
* & * & * & * & P_{i 55}
\end{array}\right]\left[\begin{array}{c}
A_{i} x(t)+B_{i} x(t-h(t))+D_{i} \omega(t)+L_{i} u \\
x(t)-x\left(t-h_{1}\right) \\
x\left(t-h_{1}\right)-x\left(t-h_{m}\right) \\
A_{i} x(t)-\dot{x}(t)+B_{i} x(t-h(t))+D_{i} \omega(t)+L_{i} u
\end{array}\right] \\
& -2\left[\begin{array}{c}
A_{i} x(t)+B_{i} x(t-h(t))+D_{i} \omega(t)+L_{i} u \\
x(t)-x\left(t-h_{1}\right) \\
x\left(t-h_{1}\right)-x\left(t-h_{m}\right) \\
x\left(t-h_{m}\right)-x\left(t-h_{2}\right)
\end{array}\right]^{T}\left[\begin{array}{c}
P_{i 15} \\
P_{i 25} \\
P_{i 35} \\
P_{i 45}
\end{array}\right] u+\eta^{T}(t)\left(\sum_{j=1}^{N} \lambda_{i j} P_{j}\right) \eta(t) .
\end{aligned}
$$

Taking into account the situation that the information of transition probabilities are not accessible completely, due to $\sum_{j=1}^{N} \lambda_{i j}=0$, the following equations hold for arbitrary appropriate matrices $W_{i}=W_{i}^{T}$ are satisfied

$$
-\eta^{T}(t)\left(\sum_{j=1}^{N} \lambda_{i j} W_{i}\right) \eta(t)=0
$$

Hence,

$$
\begin{aligned}
& L V_{1}\left(t, x_{t}\right)=2\left[\eta^{T}(t) u^{T}\right]\left[\begin{array}{ccccc}
P_{i 11} & P_{i 12} & P_{i 13} & P_{i 14} & P_{i 15} \\
* & P_{i 22} & P_{i 23} & P_{i 24} & P_{i 25} \\
* & * & P_{i 33} & P_{i 34} & P_{i 35} \\
* & * & * & P_{i 44} & P_{i 45} \\
* & * & * & * & P_{i 55}
\end{array}\right]\left[\begin{array}{c}
A_{i} x(t)+B_{i} x(t-h(t))+D_{i} \omega(t) L_{i} u \\
x(t)-x\left(t-h_{1}\right) \\
x\left(t-h_{1}\right)-x\left(t-h_{m}\right) \\
x\left(t-h_{m}\right)-x\left(t-h_{2}\right) \\
A_{i} x(t)-\dot{x}(t)+B_{i} x(t-h(t)) \\
+D_{i} \omega(t)+L_{i} u
\end{array}\right), \\
& -2\left[\begin{array}{c}
A_{i} x(t)+B_{i} x(t-h(t))+D_{i} \omega(t)+L_{i} u \\
x(t)-x\left(t-h_{1}\right) \\
x\left(t-h_{1}\right)-x\left(t-h_{m}\right) \\
x\left(t-h_{m}\right)-x\left(t-h_{2}\right)
\end{array}\right]^{T}\left[\begin{array}{c}
P_{i 15} \\
P_{i 25} \\
P_{i 35} \\
P_{i 45}
\end{array}\right] u+\eta^{T}(t)\left(\sum_{j=1}^{N} \lambda_{i j}\left(P_{j}-W_{i}\right)\right) \eta(t) \text {, } \\
& L V_{2}\left(t, x_{t}\right)=x^{T}(t) Q_{1} x(t)+e^{-\alpha h_{1}} x^{T}\left(t-h_{1}\right)\left[Q_{2}-Q_{1}\right] x\left(t-h_{1}\right)+e^{-\alpha h_{m}} x^{T}\left(t-h_{m}\right)\left[Q_{3}-Q_{2}\right] \\
& \cdot x\left(t-h_{m}\right)-e^{-\alpha h_{2}} x^{T}\left(t-h_{2}\right) Q_{3} x\left(t-h_{2}\right)-\alpha V_{2} \text {, } \\
& L V_{3}\left(t, x_{t}\right)=x^{T}(t)\left[h_{1}^{2} R_{1}+\left(h_{m}-h_{1}\right)^{2} R_{2}+\left(h_{2}-h_{m}\right)^{2} R_{3}\right] x(t)-h_{1} \int_{t-h_{1}}^{t} e^{\alpha(s-t)} x^{T}(s) R_{1} x(s) \mathrm{d} s \\
& -\left(h_{m}-h_{1}\right) \int_{t-h_{m}}^{t-h_{1}} e^{\alpha(s-t)} x^{T}(s) R_{2} x(s) \mathrm{d} s-\left(h_{2}-h_{m}\right) \int_{t-h_{2}}^{t-h_{m}} e^{\alpha(s-t)} x^{T}(s) R_{3} x(s) \mathrm{d} s-\alpha V_{3} .
\end{aligned}
$$


Based on Lemma $2, L V_{3}\left(t, x_{t}\right)$ can be rewritten as

$$
\begin{aligned}
L V_{3}\left(t, x_{t}\right) \leq & x^{T}(t)\left[h_{1}^{2} R_{1}+\left(h_{m}-h_{1}\right)^{2} R_{2}+\left(h_{2}-h_{m}\right)^{2} R_{3}\right] x(t)-e^{-\alpha h_{1}}\left(\int_{t-h_{1}}^{t} x(s) \mathrm{d} s\right)^{T} R_{1} \\
& \cdot\left(\int_{t-h_{1}}^{t} x(s) \mathrm{d} s\right)-e^{-\alpha h_{m}}\left(\int_{t-h_{m}}^{t-h_{1}} x(s) \mathrm{d} s\right)^{T} R_{2}\left(\int_{t-h_{m}}^{t-h_{1}} x(s) \mathrm{d} s\right) \\
& -e^{-\alpha h_{2}}\left(\int_{t-h_{2}}^{t-h_{m}} x(s) \mathrm{d} s\right)^{T} R_{3}\left(\int_{t-h_{2}}^{t-h_{m}} x(s) \mathrm{d} s\right)-\alpha V_{3} .
\end{aligned}
$$

So, according to Lemma 3, we have

$$
\begin{aligned}
& L V_{4}\left(t, x_{t}\right) \leq \dot{x}^{T}(t)\left[h_{1}^{2} R_{4}\right] \dot{x}(t)-e^{-\alpha h_{1}} \cdot h_{1} \int_{t-h_{1}}^{t} \dot{x}^{T}(s) R_{4} \dot{x}(s) \mathrm{d} s-\alpha V_{4} \\
& \leq \dot{x}^{T}(t)\left[h_{1}^{2} R_{4}\right] \dot{x}(t)-e^{-\alpha h_{1}}\left\{\left[x(t)-x\left(t-h_{1}\right)\right]^{T} R_{4}\left[x(t)-x\left(t-h_{1}\right)\right]\right. \\
& +\left[x(t)+x\left(t-h_{1}\right)-\frac{2}{h_{1}} \int_{t-h_{1}}^{t} x(s) \mathrm{d} s\right]^{T}\left(3 R_{4}\right)\left[x(t)+x\left(t-h_{1}\right)-\frac{2}{h_{1}} \int_{t-h_{1}}^{t} x(s) \mathrm{d} s\right] \\
& +\left[x(t)-x\left(t-h_{1}\right)+\frac{6}{h_{1}} \int_{t-h_{1}}^{t} x(s) \mathrm{d} s-\frac{12}{h_{1}^{2}} \int_{t-h_{1}}^{t} \int_{u}^{t} x(s) \mathrm{d} s \mathrm{~d} u\right]\left(5 R_{4}\right) \\
& \left.\cdot\left[x(t)-x\left(t-h_{1}\right)+\frac{6}{h_{1}} \int_{t-h_{1}}^{t} x(s) \mathrm{d} s-\frac{12}{h_{1}^{2}} \int_{t-h_{1}}^{t} \int_{u}^{t} x(s) \mathrm{d} s \mathrm{~d} u\right]\right\}-\alpha V_{4} \text {, } \\
& L V_{5}\left(t, x_{t}\right) \leq \dot{x}^{T}(t)\left[\left(h_{m}-h_{1}\right)^{2} R_{5}+\left(h_{2}-h_{m}\right)^{2} R_{6}\right] \dot{x}(t)-e^{-\alpha h_{m}}\left(h_{m}-h_{1}\right) \int_{t-h_{m}}^{t-h_{1}} \dot{x}^{T}(s) R_{5} \dot{x}(s) \mathrm{d} s \\
& -e^{-\alpha h_{2}}\left(h_{2}-h_{m}\right) \int_{t-h_{2}}^{t-h_{m}} \dot{x}^{T}(s) R_{6} \dot{x}(s) \mathrm{d} s-\alpha V_{5} .
\end{aligned}
$$

When $h(t) \in\left(h_{1}, h_{m}\right)$, based on the Lemmas 3 and 4 , we have

$$
\begin{aligned}
& -\left(h_{m}-h_{1}\right) \int_{t-h_{m}}^{t-h_{1}} \dot{x}^{T}(s) R_{5} \dot{x}(s) \mathrm{d} s \\
= & -\left(h_{m}-h_{1}\right) \int_{t-h_{m}}^{t-h(t)} \dot{x}^{T}(s) R_{5} \dot{x}(s) \mathrm{d} s-\left(h_{m}-h_{1}\right) \int_{t-h(t)}^{t-h_{1}} \dot{x}^{T}(s) R_{5} \dot{x}(s) \mathrm{d} s \\
\leq & -\xi_{1}^{T}(t)\left[\frac{1}{\left(h_{m}-h(t)\right) /\left(h_{m}-h_{1}\right)} \Gamma_{1}^{T} \widetilde{R}_{5} \Gamma_{1}+\frac{1}{\left(h(t)-h_{1}\right) /\left(h_{m}-h_{1}\right)} \Gamma_{2}^{T} \widetilde{R}_{5} \Gamma_{2}\right] \xi_{1}(t) \\
\leq & -\xi_{1}^{T}(t)\left[\begin{array}{c}
\Gamma_{1} \\
\Gamma_{2}
\end{array}\right]^{T}\left[\begin{array}{cc}
\widetilde{R}_{5} & X \\
* & \widetilde{R}_{5}
\end{array}\right]\left[\begin{array}{c}
\Gamma_{1} \\
\Gamma_{2}
\end{array}\right] \xi_{1}(t),
\end{aligned}
$$


Journal of Mathematics

7

where

$$
\begin{aligned}
& \xi_{1}(t)=\left[x^{T}(t) \dot{x}^{T}(t) x^{T}\left(t-h_{1}\right) x^{T}(t-h(t)) x^{T}\left(t-h_{m}\right) x^{T}\left(t-h_{2}\right)\left(\int_{t-h_{1}}^{t} x(s) \mathrm{d} s\right)^{T}\left(\int_{t-h_{m}}^{t-h_{1}} x(s) \mathrm{d} s\right)^{T}\right. \\
& \cdot\left(\int_{t-h_{2}}^{t-h_{m}} x(s) \mathrm{d} s\right)^{T}\left(\frac{1}{h_{m}-h(t)} \int_{t-h_{m}}^{t-h(t)} x(s) \mathrm{d} s\right)^{T}\left(\frac{1}{h(t)-h_{1}} \int_{t-h(t)}^{t-h_{1}} x(s) \mathrm{d} s\right)^{T}\left(\frac{1}{\left(h_{m}-h(t)\right)^{2}} \int_{t-h_{m}}^{t-h(t)} \int_{u}^{t-h(t)} x(s) \mathrm{d} s \mathrm{~d} u\right)^{T} \\
& \left.\cdot\left(\frac{1}{\left(h(t)-h_{1}\right)^{2}} \int_{t-h(t)}^{t-h_{1}} \int_{u}^{t-h_{1}} x(s) \mathrm{d} s \mathrm{~d} u\right)^{T}\left(\frac{1}{h_{1}^{2}} \int_{t-h_{1}}^{t} \int_{u}^{t} x(s) \mathrm{d} s \mathrm{~d} u\right)^{T}\left(\frac{1}{\left(h_{2}-h_{m}\right)^{2}} \int_{t-h_{2}}^{t-h_{m}} \int_{u}^{t-h_{m}} x(s) \mathrm{d} s \mathrm{~d} u\right)^{T} \omega^{T}(t) u^{T}\right]^{T}, \\
& \chi_{1}=\left[\begin{array}{lllllllllllllllll}
0 & 0 & 0 & I & -I & 0 & 0 & 0 & 0 & 0 & 0 & 0 & 0 & 0 & 0 & 0 & 0
\end{array}\right] \text {, } \\
& \chi_{2}=\left[\begin{array}{lllllllllllllllll}
0 & 0 & 0 & I & I & 0 & 0 & 0 & 0 & -2 I & 0 & 0 & 0 & 0 & 0 & 0 & 0
\end{array}\right], \\
& \chi_{3}=\left[\begin{array}{lllllllllllllllll}
0 & 0 & 0 & I & -I & 0 & 0 & 0 & 0 & 6 I & 0 & -12 I & 0 & 0 & 0 & 0 & 0
\end{array}\right], \\
& \chi_{4}=\left[\begin{array}{lllllllllllllllll}
0 & 0 & I & -I & 0 & 0 & 0 & 0 & 0 & 0 & 0 & 0 & 0 & 0 & 0 & 0 & 0
\end{array}\right], \\
& \chi_{5}=\left[\begin{array}{lllllllllllllllll}
0 & 0 & I & I & 0 & 0 & 0 & 0 & 0 & 0 & -2 I & 0 & 0 & 0 & 0 & 0 & 0
\end{array}\right], \\
& \chi_{6}=\left[\begin{array}{lllllllllllllllll}
0 & 0 & I & -I & 0 & 0 & 0 & 0 & 0 & 0 & 6 I & 0 & -12 I & 0 & 0 & 0 & 0
\end{array}\right], \\
& \Gamma_{1}=\left[\begin{array}{l}
\chi_{1} \\
\chi_{2} \\
\chi_{3}
\end{array}\right] \\
& \Gamma_{2}=\left[\begin{array}{l}
\chi_{4} \\
\chi_{5} \\
\chi_{6}
\end{array}\right], \\
& \widetilde{R}_{5}=\left[\begin{array}{ccc}
R_{5} & 0 & 0 \\
0 & 3 R_{5} & 0 \\
0 & 0 & 5 R_{5}
\end{array}\right] \text {, } \\
& {\left[\begin{array}{cc}
\widetilde{R}_{5} & X \\
* & \widetilde{R}_{5}
\end{array}\right]>0}
\end{aligned}
$$


Using Lemma 3, we further have

$$
\begin{aligned}
- & \left(h_{2}-h_{m}\right) \int_{t-h_{2}}^{t-h_{m}} \dot{x}^{T}(s) R_{6} \dot{x}(s) \mathrm{d} s \\
\leq & -\left\{9 x^{T}\left(t-h_{m}\right) R_{6} x\left(t-h_{m}\right)-6 x^{T}\left(t-h_{m}\right) R_{6} x\left(t-h_{2}\right)+9 x^{T}\left(t-h_{2}\right) R_{6} x\left(t-h_{2}\right)+\frac{48}{h_{2}-h_{m}}\right. \\
& \cdot x^{T}\left(t-h_{m}\right) R_{6}\left(\int_{t-h_{2}}^{t-h_{m}} x(s) \mathrm{d} s\right)-\frac{120}{\left(h_{2}-h_{m}\right)^{2}} x^{T}\left(t-h_{m}\right) R_{6}\left(\int_{t-h_{2}}^{t-h_{m}} \int_{u}^{t-h_{m}} x(s) \mathrm{d} s \mathrm{~d} u\right)-\frac{72}{h_{2}-h_{m}} \\
& \cdot x^{T}\left(t-h_{2}\right) R_{6}\left(\int_{t-h_{2}}^{t-h_{m}} x(s) \mathrm{d} s\right)+\frac{120}{\left(h_{2}-h_{m}\right)^{2}} x^{T}\left(t-h_{2}\right) R_{6}\left(\int_{t-h_{2}}^{t-h_{m}} \int_{u}^{t-h_{m}} x(s) \mathrm{d} s \mathrm{~d} u\right)+\frac{192}{\left(h_{2}-h_{m}\right)^{2}} \\
& \cdot\left(\int_{t-h_{2}}^{t-h_{m}} x(s) \mathrm{d} s\right)^{T} R_{6}\left(\int_{t-h_{2}}^{t-h_{m}} x(s) \mathrm{d} s\right)^{-} \frac{720}{\left(h_{2}-h_{m}\right)^{3}}\left(\int_{t-h_{2}}^{t-h_{m}} x(s) \mathrm{d} s\right)^{T} R_{6}\left(\int_{t-h_{2}}^{t-h_{m}} \int_{u}^{t-h_{m}} x(s) \mathrm{d} s \mathrm{~d} u\right) \\
& \left.+\frac{720}{\left(h_{2}-h_{m}\right)^{4}}\left(\int_{t-h_{2}}^{t-h_{m}} \int_{u}^{t-h_{m}} x(s) \mathrm{d} s \mathrm{~d} u\right)^{T} R_{6}\left(\int_{t-h_{2}}^{t-h_{m}} \int_{u}^{t-h_{m}} x(s) \mathrm{d} s \mathrm{~d} u\right)\right\} .
\end{aligned}
$$

Thus, $L V_{5}\left(t, x_{t}\right)$ can be acquired as

$$
\begin{aligned}
& L V_{5}\left(t, x_{t}\right) \leq \dot{x}^{T}(t)\left[\left(h_{m}-h_{1}\right)^{2} R_{5}+\left(h_{2}-h_{m}\right)^{2} R_{6}\right] \dot{x}(t)-e^{-\alpha h_{m}} \xi_{1}^{T}(t)\left[\begin{array}{c}
\Gamma_{1} \\
\Gamma_{2}
\end{array}\right]^{T}\left[\begin{array}{cc}
\widetilde{R}_{5} & X \\
* & \widetilde{R}_{5}
\end{array}\right]\left[\begin{array}{l}
\Gamma_{1} \\
\Gamma_{2}
\end{array}\right] \xi_{1}(t) \\
& -e^{-\alpha h_{2}}\left\{9 x^{T}\left(t-h_{m}\right) R_{6} x\left(t-h_{m}\right)-6 x^{T}\left(t-h_{m}\right) R_{6} x\left(t-h_{2}\right)+9 x^{T}\left(t-h_{2}\right) R_{6} x\left(t-h_{2}\right)\right. \\
& +\frac{48}{h_{2}-h_{m}} x^{T}\left(t-h_{m}\right) R_{6}\left(\int_{t-h_{2}}^{t-h_{m}} x(s) \mathrm{d} s\right)-\frac{120}{\left(h_{2}-h_{m}\right)^{2}} x^{T}\left(t-h_{m}\right) R_{6}\left(\int_{t-h_{2}}^{t-h_{m}} \int_{u}^{t-h_{m}} x(s) \mathrm{d} s \mathrm{~d} u\right) \\
& -\frac{72}{h_{2}-h_{m}} x^{T}\left(t-h_{2}\right) R_{6}\left(\int_{t-h_{2}}^{t-h_{m}} x(s) \mathrm{d} s\right)+\frac{120}{\left(h_{2}-h_{m}\right)^{2}} x^{T}\left(t-h_{2}\right) R_{6}\left(\int_{t-h_{2}}^{t-h_{m}} \int_{u}^{t-h_{m}} x(s) \mathrm{d} s \mathrm{~d} u\right) \\
& +\frac{192}{\left(h_{2}-h_{m}\right)^{2}}\left(\int_{t-h_{2}}^{t-h_{m}} x(s) \mathrm{d} s\right)^{T} R_{6}\left(\int_{t-h_{2}}^{t-h_{m}} x(s) \mathrm{d} s\right)-\frac{720}{\left(h_{2}-h_{m}\right)^{3}}\left(\int_{t-h_{2}}^{t-h_{m}} x(s) \mathrm{d} s\right)^{T} R_{6} \\
& \cdot\left(\int_{t-h_{2}}^{t-h_{m}} \int_{u}^{t-h_{m}} x(s) \mathrm{d} s \mathrm{~d} u\right)+\frac{720}{\left(h_{2}-h_{m}\right)^{4}}\left(\int_{t-h_{2}}^{t-h_{m}} \int_{u}^{t-h_{m}} x(s) \mathrm{d} s \mathrm{~d} u\right)^{T} \\
& \cdot R_{6}\left(\int_{t-h_{2}}^{t-h_{m}} \int_{u}^{t-h_{m}} x(s) \mathrm{d} s \mathrm{~d} u\right)-\alpha V_{5} .
\end{aligned}
$$

Meanwhile, for any matrices $M_{1}$ and $M_{2}$ with appropriate dimension, the following equation is true:

$$
\begin{aligned}
& 2\left[x^{T}(t) M_{1}+\dot{x}^{T}(t) M_{2}\right] \\
& \quad \cdot\left[A_{i} x(t)-\dot{x}(t)+B_{i} x(t-h(t))+D_{i} \omega(t)+L_{i} u\right]=0 .
\end{aligned}
$$

Combining equations (25)-(37), we can obtain

$$
\begin{aligned}
& L V\left(t, x_{t}\right)+\alpha V\left(t, x_{t}\right)-\frac{\alpha}{\omega_{m}^{2}} \omega^{T}(t) \omega(t) \leq \xi_{1}^{T}(t) \\
& \cdot\left(\Psi_{i}+\Phi_{1}\right) \xi_{1}(t)+\eta^{T}(t)\left(\sum_{j \in U_{u k}^{i}} \lambda_{i j}\left(P_{j}-W_{i}\right)\right) \eta(t),
\end{aligned}
$$


where $\Psi_{i}, \Phi_{1}$ are the same as defined in Theorem 1. Using the S-procedure in [4], one can see that this condition is implied by the existence of a non-negative scalar $\varepsilon>0$ such that

$$
\begin{aligned}
& L V\left(t, x_{t}\right)+\alpha V\left(t, x_{t}\right)-\frac{\alpha}{\omega_{m}^{2}} \omega^{T}(t) \omega(t) \leq \xi_{1}^{T}(t)\left(\Psi_{i}+\Phi_{1}\right) \xi_{1}(t)+\eta^{T}(t)\left(\sum_{j \in U_{u k}^{i}} \lambda_{i j}\left(P_{j}-W_{i}\right)\right) \eta(t) \\
& +\varepsilon\left\{\left[E_{i a} x(t)+E_{i b} x(t-h(t))+E_{i d} \omega(t)\right]^{T}\left[E_{i a} x(t)+E_{i b} x(t-h(t))+E_{i d} \omega(t)\right]-u^{T} u\right\}<0,
\end{aligned}
$$

for all $\xi_{1}(t) \neq 0$. By using Lemma 5 , the matrix inequalities (21), (23), and (24) imply (41).

Similarly, when $h(t)=h_{1}$ or $h(t)=h_{m}$, inequality (35) can be reduced to the following two inequalities,

$$
\begin{aligned}
& -\left(h_{m}-h_{1}\right) \int_{t-h_{m}}^{t-h_{1}} \dot{x}^{T}(s) R_{5} \dot{x}(s) \mathrm{d} s \\
= & -\left(h_{m}-h(t)\right) \int_{t-h_{m}}^{t-h(t)} \dot{x}^{T}(s) R_{5} \dot{x}(s) \mathrm{d} s \\
\leq & -\left\{\left[x(t-h(t))-x\left(t-h_{m}\right)\right]^{T} R_{5}\left[x(t-h(t))-x\left(t-h_{m}\right)\right]+\left[x(t-h(t))+x\left(t-h_{m}\right)\right.\right. \\
& \left.-\frac{2}{h_{m}-h(t)} \int_{t-h_{m}}^{t-h(t)} x(s) \mathrm{d} s\right]^{T}\left(3 R_{5}\right)\left[x(t-h(t))+x\left(t-h_{m}\right)-\frac{2}{h_{m}-h(t)} \int_{t-h_{m}}^{t-h(t)} x(s) \mathrm{d} s\right] \\
& +\left[x(t-h(t))-x\left(t-h_{m}\right)+\frac{6}{h_{m}-h(t)} \int_{t-h_{m}}^{t-h(t)} x(s) \mathrm{d} s-\frac{12}{\left(h_{m}-h(t)\right)^{2}} \int_{t-h_{m}}^{t-h(t)} \int_{u}^{t-h(t)} x(s) \mathrm{d} s \mathrm{~d} u\right]^{T} \\
& \cdot\left(5 R_{5}\right)\left[x(t-h(t))-x\left(t-h_{m}\right)+\frac{6}{h_{m}-h(t)} \int_{t-h_{m}}^{t-h(t)} x(s) \mathrm{d} s-\frac{12}{\left(h_{m}-h(t)\right)^{2}} \int_{t-h_{m}}^{t-h(t)} \int_{u}^{t-h(t)} x(s) \mathrm{d} s \mathrm{~d} u\right] .
\end{aligned}
$$

Combining Equations (25)-(39) and (42), we can obtain

$$
\begin{aligned}
& L V\left(t, x_{t}\right)+\alpha V\left(t, x_{t}\right)-\frac{\alpha}{\omega_{m}^{2}} \omega^{T}(t) \omega(t) \\
& \quad \leq \xi_{1}^{T}(t)\left(\widehat{\Psi}_{i}+\Phi_{2}\right) \xi_{1}(t)+\eta^{T}(t)\left(\sum_{j \in U_{u k}^{i}} \lambda_{i j}\left(P_{j}-W_{i}\right)\right) \eta(t),
\end{aligned}
$$

where $\widehat{\Psi}_{i}, \Phi_{2}$ are the same as defined in Theorem 1. Using the $S$-procedure in [4], one can see that this condition is implied by the existence of a non-negative scalar $\varepsilon>0$ such that

$$
\begin{aligned}
& L V\left(t, x_{t}\right)+\alpha V\left(t, x_{t}\right)-\frac{\alpha}{\omega_{m}^{2}} \omega^{T}(t) \omega(t) \leq \xi_{1}^{T}(t)\left(\widehat{\Psi}_{i}+\Phi_{2}\right) \xi_{1}(t)+\eta^{T}(t)\left(\sum_{j \in U_{u k}^{i}} \lambda_{i j}\left(P_{j}-W_{i}\right)\right) \eta(t) \\
& \quad+\varepsilon\left\{\left[E_{i a} x(t)+E_{i b} x(t-h(t))+E_{i d} \omega(t)\right]^{T}\left[E_{i a} x(t)+E_{i b} x(t-h(t))+E_{i d} \omega(t)\right]-u^{T} u\right\}<0,
\end{aligned}
$$




$$
\begin{aligned}
& -\left(h_{m}-h_{1}\right) \int_{t-h_{m}}^{t-h_{1}} \dot{x}^{T}(s) R_{5} \dot{x}(s) \mathrm{d} s \\
= & -\left(h(t)-h_{1}\right) \int_{t-h(t)}^{t-h_{1}} \dot{x}^{T}(s) R_{5} \dot{x}(s) \mathrm{d} s \\
\leq & -\left\{\left[x\left(t-h_{1}\right)-x(t-h(t))\right]^{T} R_{5}\left[x\left(t-h_{1}\right)-x(t-h(t))\right]+\left[x\left(t-h_{1}\right)+x(t-h(t))\right.\right. \\
& \left.-\frac{2}{h(t)-h_{1}} \int_{t-h(t)}^{t-h_{1}} x(s) \mathrm{d} s\right]\left(3 R_{5}\right)\left[x\left(t-h_{1}\right)+x(t-h(t))-\frac{2}{h(t)-h_{1}} \int_{t-h(t)}^{t-h_{1}} x(s) \mathrm{d} s\right] \\
& +\left[x\left(t-h_{1}\right)-x(t-h(t))+\frac{6}{h(t)-h_{1}} \int_{t-h(t)}^{t-h_{1}} x(s) \mathrm{d} s-\frac{12}{\left(h(t)-h_{1}\right)^{2}} \int_{t-h(t)}^{t-h_{1}} \int_{u}^{t-h_{1}} x(s) \mathrm{d} s \mathrm{~d} u\right]^{T} \\
& \left.\cdot\left(5 R_{5}\right)\left[x\left(t-h_{1}\right)-x(t-h(t))+\frac{6}{h(t)-h_{1}} \int_{t-h(t)}^{t-h_{1}} x(s) \mathrm{d} s-\frac{12}{\left(h(t)-h_{1}\right)^{2}} \int_{t-h(t)}^{t-h_{1}} \int_{u}^{t-h_{1}} x(s) \mathrm{d} s \mathrm{~d} u\right]\right\} .
\end{aligned}
$$

Combining Equations (25)-(39) and (45), we can obtain

$$
\begin{aligned}
& L V\left(t, x_{t}\right)+\alpha V\left(t, x_{t}\right)-\frac{\alpha}{\omega_{m}^{2}} \omega^{T}(t) \omega(t) \leq \xi_{1}^{T}(t)\left(\widehat{\Psi}_{i}+\Phi_{3}\right) \xi_{1}(t) \\
& \quad+\eta^{T}(t)\left(\sum_{j \in U_{u k}^{i}} \lambda_{i j}\left(P_{j}-W_{i}\right)\right) \eta(t),
\end{aligned}
$$

$$
\begin{aligned}
& L V\left(t, x_{t}\right)+\alpha V\left(t, x_{t}\right)-\frac{\alpha}{\omega_{m}^{2}} \omega^{T}(t) \omega(t) \leq \xi_{1}^{T}(t)\left(\widehat{\Psi}_{i}+\Phi_{3}\right) \xi_{1}(t)+\eta^{T}(t)\left(\sum_{j \in U_{u k}^{i}} \lambda_{i j}\left(P_{j}-W_{i}\right)\right) \eta(t) \\
& \quad+\varepsilon\left\{\left[E_{i a} x(t)+E_{i b} x(t-h(t))+E_{i d} \omega(t)\right]^{T}\left[E_{i a} x(t)+E_{i b} x(t-h(t))+E_{i d} \omega(t)\right]-u^{T} u\right\}<0,
\end{aligned}
$$

for all $\xi_{1}(t) \neq 0$. By using Lemma 5 , the matrix inequalities (21), (23), and (24) imply (47). where $\widehat{\Psi}_{i}, \Phi_{3}$ are the same as defined in Theorem 1. Using the $S$-procedure in [4], one can see that this condition is implied by the existence of a non-negative scalar $\varepsilon>0$ such that
It should be noted that $\Psi_{i}+\Phi_{1}<0, \widehat{\Psi}_{i}+\Phi_{2}<0, \widehat{\Psi}_{i}+$ $\Phi_{3}<0$ according to inequalities (21), (23), and (24), which implies that $L V\left(t, x_{t}\right)+\alpha V\left(t, x_{t}\right)-\left(\alpha / \omega_{m}^{2}\right) \omega^{T}(t) \omega(t)<0$. 
When $h(t) \in\left(h_{m}, h_{2}\right)$, the last two integral terms of $L V_{5}\left(t, x_{t}\right)$ are handled in the following way based on Lemmas 3 and 4:

$$
\begin{aligned}
& -\left(h_{m}-h_{1}\right) \int_{t-h_{m}}^{t-h_{1}} \dot{x}^{T}(s) R_{5} \dot{x}(s) \mathrm{d} s \\
\leq & -\left\{9 x^{T}\left(t-h_{1}\right) R_{5} x\left(t-h_{1}\right)-6 x^{T}\left(t-h_{1}\right) R_{5} x\left(t-h_{m}\right)+9 x^{T}\left(t-h_{m}\right) R_{5} x\left(t-h_{m}\right)\right. \\
& +\frac{48}{h_{m}-h_{1}} x^{T}\left(t-h_{1}\right) R_{6}\left(\int_{t-h_{m}}^{t-h_{1}} x(s) \mathrm{d} s\right)-\frac{120}{\left(h_{m}-h_{1}\right)^{2}} x^{T}\left(t-h_{1}\right) R_{5}\left(\int_{t-h_{m}}^{t-h_{1}} \int_{u}^{t-h_{1}} x(s) \mathrm{d} s \mathrm{~d} u\right) \\
& -\frac{72}{h_{m}-h_{1}} x^{T}\left(t-h_{m}\right) R_{5}\left(\int_{t-h_{m}}^{t-h_{1}} x(s) \mathrm{d} s\right)+\frac{120}{\left(h_{m}-h_{1}\right)^{2}} x^{T}\left(t-h_{m}\right) R_{5}\left(\int_{t-h_{m}}^{t-h_{1}} \int_{u}^{t-h_{1}} x(s) \mathrm{d} s \mathrm{~d} u\right) \\
& +\frac{192}{\left(h_{m}-h_{1}\right)^{2}}\left(\int_{t-h_{m}}^{t-h_{1}} x(s) \mathrm{d} s\right)^{T} R_{5}\left(\int_{t-h_{m}}^{t-h_{1}} x(s) \mathrm{d} s\right)-\frac{720}{\left(h_{m}-h_{1}\right)^{3}}\left(\int_{t-h_{m}}^{t-h_{1}} x(s) \mathrm{d} s\right)^{T} R_{5} \\
& \left.\cdot\left(\int_{t-h_{m}}^{t-h_{1}} \int_{u}^{t-h_{1}} x(s) \mathrm{d} s \mathrm{~d} u\right)+\frac{720}{\left(h_{m}-h_{1}\right)^{4}}\left(\int_{t-h_{m}}^{t-h_{1}} \int_{u}^{t-h_{1}} x(s) \mathrm{d} s \mathrm{~d} u\right)^{T} R_{5}\left(\int_{t-h_{m}}^{t-h_{1}} \int_{u}^{t-h_{1}} x(s) \mathrm{d} s \mathrm{~d} u\right)\right\} .
\end{aligned}
$$

Using the same method to deal with the following integral inequality, we further have

$$
\begin{aligned}
& -\left(h_{2}-h_{m}\right) \int_{t-h_{2}}^{t-h_{m}} \dot{x}^{T}(s) R_{6} \dot{x}(s) \mathrm{d} s \\
= & -\left(h_{2}-h_{m}\right) \int_{t-h_{2}}^{t-h(t)} \dot{x}^{T}(s) R_{6} \dot{x}(s) \mathrm{d} s-\left(h_{2}-h_{m}\right) \int_{t-h(t)}^{t-h_{m}} \dot{x}^{T}(s) R_{6} \dot{x}(s) \mathrm{d} s \\
\leq & -\xi_{2}^{T}(t)\left[\frac{1}{\left(h_{2}-h(t)\right) /\left(h_{2}-h_{m}\right)} \Gamma_{3}^{T} \widetilde{R}_{6} \Gamma_{3}+\frac{1}{\left(h(t)-h_{m}\right) /\left(h_{2}-h_{m}\right)} \Gamma_{4}^{T} \widetilde{R}_{6} \Gamma_{4}\right] \xi_{2}(t) \\
\leq & -\xi_{2}^{T}(t)\left[\begin{array}{c}
\Gamma_{3} \\
\Gamma_{4}
\end{array}\right]^{T}\left[\begin{array}{cc}
\widetilde{R}_{6} & Y \\
* & \widetilde{R}_{6}
\end{array}\right]\left[\begin{array}{c}
\Gamma_{3} \\
\Gamma_{4}
\end{array}\right] \xi_{2}(t),
\end{aligned}
$$


12

Journal of Mathematics

where

$$
\begin{aligned}
& \xi_{2}(t)=\left[x^{T}(t) \dot{x}^{T}(t) x^{T}\left(t-h_{1}\right) x^{T}(t-h(t)) x^{T}\left(t-h_{m}\right) x^{T}\left(t-h_{2}\right)\left(\int_{t-h_{1}}^{t} x(s) \mathrm{d} s\right)^{T}\left(\int_{t-h_{m}}^{t-h_{1}} x(s) \mathrm{d} s\right)^{T}\right. \\
& \cdot\left(\int_{t-h_{2}}^{t-h_{m}} x(s) \mathrm{d} s\right)^{T}\left(\frac{1}{h_{2}-h(t)} \int_{t-h_{2}}^{t-h(t)} x(s) \mathrm{d} s\right)^{T}\left(\frac{1}{h(t)-h_{m}} \int_{t-h(t)}^{t-h_{m}} x(s) \mathrm{d} s\right)^{T}\left(\frac{1}{\left(h_{2}-h(t)\right)^{2}} \int_{t-h_{2}}^{t-h(t)} \int_{u}^{t-h(t)} x(s) \mathrm{d} s \mathrm{~d} u\right)^{T} \\
& \left.\cdot\left(\frac{1}{\left(h(t)-h_{m}\right)^{2}} \int_{t-h(t)}^{t-h_{m}} \int_{u}^{t-h_{m}} x(s) \mathrm{d} s \mathrm{~d} u\right)^{T}\left(\frac{1}{h_{1}^{2}} \int_{t-h_{1}}^{t} \int_{u}^{t} x(s) \mathrm{d} s \mathrm{~d} u\right)^{T}\left(\frac{1}{\left(h_{m}-h_{1}\right)^{2}} \int_{t-h_{m}}^{t-h_{1}} \int_{u}^{t-h_{1}} x(s) \mathrm{d} s \mathrm{~d} u\right)^{T} \omega^{T}(t) u^{T}\right]^{T}, \\
& \chi_{7}=\left[\begin{array}{lllllllllllllllll}
0 & 0 & 0 & I & 0 & -I & 0 & 0 & 0 & 0 & 0 & 0 & 0 & 0 & 0 & 0 & 0
\end{array}\right] \text {, } \\
& \chi_{8}=\left[\begin{array}{lllllllllllllllll}
0 & 0 & 0 & I & 0 & I & 0 & 0 & 0 & -2 I & 0 & 0 & 0 & 0 & 0 & 0 & 0
\end{array}\right], \\
& \chi_{9}=\left[\begin{array}{lllllllllllllllll}
0 & 0 & 0 & I & 0 & -I & 0 & 0 & 0 & 6 I & 0 & -12 I & 0 & 0 & 0 & 0 & 0
\end{array}\right], \\
& \chi_{10}=\left[\begin{array}{lllllllllllllllll}
0 & 0 & 0 & -I & I & 0 & 0 & 0 & 0 & 0 & 0 & 0 & 0 & 0 & 0 & 0 & 0
\end{array}\right], \\
& \chi_{11}=\left[\begin{array}{lllllllllllllllll}
0 & 0 & 0 & I & I & 0 & 0 & 0 & 0 & 0 & -2 I & 0 & 0 & 0 & 0 & 0 & 0
\end{array}\right], \\
& \chi_{12}=\left[\begin{array}{lllllllllllllllll}
0 & 0 & 0 & -I & I & 0 & 0 & 0 & 0 & 0 & 6 I & 0 & -12 I & 0 & 0 & 0 & 0
\end{array}\right], \\
& \Gamma_{3}=\left[\begin{array}{l}
\chi_{7} \\
\chi_{8} \\
\chi_{9}
\end{array}\right], \\
& \Gamma_{4}=\left[\begin{array}{l}
\chi_{10} \\
\chi_{11} \\
\chi_{12}
\end{array}\right], \\
& \widetilde{R}_{6}=\left[\begin{array}{ccc}
R_{6} & 0 & 0 \\
0 & 3 R_{6} & 0 \\
0 & 0 & 5 R_{6}
\end{array}\right] \text {, } \\
& {\left[\begin{array}{cc}
\widetilde{R}_{6} & Y \\
* & \widetilde{R}_{6}
\end{array}\right]>0 .}
\end{aligned}
$$


Then, $L V_{5}\left(t, x_{t}\right)$ can be rewritten as

$$
\begin{aligned}
& L V_{5}\left(t, x_{t}\right) \leq \dot{x}^{T}(t)\left[\left(h_{m}-h_{1}\right)^{2} R_{5}+\left(h_{2}-h_{m}\right)^{2} R_{6}\right] \dot{x}(t)-e^{-\alpha h_{m}}\left\{9 x^{T}\left(t-h_{1}\right) R_{5} x\left(t-h_{1}\right)\right. \\
& -6 x^{T}\left(t-h_{1}\right) R_{5} x\left(t-h_{m}\right)+9 x^{T}\left(t-h_{m}\right) R_{5} x\left(t-h_{m}\right)+\frac{48}{h_{m}-h_{1}} x^{T}\left(t-h_{1}\right) R_{5} \\
& \cdot\left(\int_{t-h_{m}}^{t-h_{1}} x(s) \mathrm{d} s\right)-\frac{120}{\left(h_{m}-h_{1}\right)^{2}} x^{T}\left(t-h_{1}\right) R_{5}\left(\int_{t-h_{m}}^{t-h_{1}} \int_{u}^{t-h_{1}} x(s) \mathrm{d} s \mathrm{~d} u\right)-\frac{72}{h_{m}-h_{1}} \\
& \cdot x^{T}\left(t-h_{m}\right) R_{5}\left(\int_{t-h_{m}}^{t-h_{1}} x(s) \mathrm{d} s\right)+\frac{120}{\left(h_{m}-h_{1}\right)^{2}} x^{T}\left(t-h_{m}\right) R_{5}\left(\int_{t-h_{m}}^{t-h_{1}} \int_{u}^{t-h_{1}} x(s) \mathrm{d} s \mathrm{~d} u\right) \\
& +\frac{192}{\left(h_{m}-h_{1}\right)^{2}}\left(\int_{t-h_{m}}^{t-h_{1}} x(s) \mathrm{d} s\right)^{T} R_{5}\left(\int_{t-h_{m}}^{t-h_{1}} x(s) \mathrm{d} s\right)-\frac{720}{\left(h_{m}-h_{1}\right)^{3}}\left(\int_{t-h_{m}}^{t-h_{1}} x(s) \mathrm{d} s\right)^{T} R_{5} \\
& \cdot\left(\int_{t-h_{m}}^{t-h_{1}} \int_{u}^{t-h_{1}} x(s) \mathrm{d} s \mathrm{~d} u\right)+\frac{720}{\left(h_{m}-h_{1}\right)^{4}}\left(\int_{t-h_{m}}^{t-h_{1}} \int_{u}^{t-h_{1}} x(s) \mathrm{d} s \mathrm{~d} u\right)^{T} R_{5} \\
& \left.\cdot\left(\int_{t-h_{m}}^{t-h_{1}} \int_{u}^{t-h_{1}} x(s) \mathrm{d} s \mathrm{~d} u\right)\right\}-e^{-\alpha h_{2}} \xi_{2}^{T}(t)\left[\begin{array}{c}
\Gamma_{3} \\
\Gamma_{4}
\end{array}\right]^{T}\left[\begin{array}{cc}
\widetilde{R}_{6} & Y \\
* & \widetilde{R}_{6}
\end{array}\right]\left[\begin{array}{c}
\Gamma_{3} \\
\Gamma_{4}
\end{array}\right] \xi_{2}(t)-\alpha V_{5} .
\end{aligned}
$$

Based on Equations (25)-(34), (39), and (51), we have $L V\left(t, x_{t}\right)+\alpha V\left(t, x_{t}\right)-\frac{\alpha}{\omega_{m}^{2}} \omega^{T}(t) \omega(t) \leq \xi_{2}^{T}(t)\left(\Psi_{i}+\Pi_{1}\right) \xi_{2}(t)$$$
+\eta^{T}(t)\left(\sum_{j \in U_{u k}^{i}} \lambda_{i j}\left(P_{j}-W_{i}\right)\right) \eta(t),
$$

$$
\begin{aligned}
& L V\left(t, x_{t}\right)+\alpha V\left(t, x_{t}\right)-\frac{\alpha}{\omega_{m}^{2}} \omega^{T}(t) \omega(t) \leq \xi_{2}^{T}(t)\left(\Psi_{i}+\Pi_{1}\right) \xi_{2}(t)+\eta^{T}(t)\left(\sum_{j \in U_{u k}^{i}} \lambda_{i j}\left(P_{j}-W_{i}\right)\right) \eta(t) \\
& \quad+\varepsilon\left\{\left[E_{i a} x(t)+E_{i b} x(t-h(t))+E_{i d} \omega(t)\right]^{T}\left[E_{i a} x(t)+E_{i b} x(t-h(t))+E_{i d} \omega(t)\right]-u^{T} u\right\}<0,
\end{aligned}
$$

for all $\xi_{2}(t) \neq 0$. By using Lemma 5 , the matrix inequalities (22)-(24) imply (53). where $\Psi_{i}, \Pi_{1}$ are the same as defined in Theorem 1. Using the $S$-procedure in [4], one can see that this condition is implied by the existence of a non-negative scalar $\varepsilon>0$ such that
When $h(t)=h_{2}$, inequality (49) can simplify the following equation: 


$$
\begin{aligned}
& -\left(h_{2}-h_{m}\right) \int_{t-h_{2}}^{t-h_{m}} \dot{x}^{T}(s) R_{6} \dot{x}(s) \mathrm{d} s \\
= & -\left(h(t)-h_{m}\right) \int_{t-h(t)}^{t-h_{m}} \dot{x}^{T}(s) R_{6} \dot{x}(s) \mathrm{d} s \\
\leq & -\left\{\left[x\left(t-h_{m}\right)-x(t-h(t))\right]^{T} R_{6}\left[x\left(t-h_{m}\right)-x(t-h(t))\right]+\left[x\left(t-h_{m}\right)+x(t-h(t))\right.\right. \\
& \left.-\frac{2}{h(t)-h_{m}} \int_{t-h(t)}^{t-h_{m}} x(s) \mathrm{d} s\right]^{T}\left(3 R_{6}\right)\left[x\left(t-h_{m}\right)+x(t-h(t))-\frac{2}{h(t)-h_{m}} \int_{t-h(t)}^{t-h_{m}} x(s) \mathrm{d} s\right] \\
& +\left[x\left(t-h_{m}\right)-x(t-h(t))+\frac{6}{h(t)-h_{m}} \int_{t-h(t)}^{t-h_{m}} x(s) \mathrm{d} s-\frac{12}{\left(h(t)-h_{m}\right)^{2}} \int_{t-h(t)}^{t-h_{m}} \int_{u}^{t-h_{m}} x(s) \mathrm{d} s\right]^{T} \\
& \left.\cdot\left(5 R_{6}\right)\left[x\left(t-h_{m}\right)-x(t-h(t))+\frac{6}{h(t)-h_{m}} \int_{t-h(t)}^{t-h_{m}} x(s) \mathrm{d} s-\frac{12}{\left(h(t)-h_{m}\right)^{2}} \int_{t-h(t)}^{t-h_{m}} \int_{u}^{t-h_{m}} x(s) \mathrm{d} s\right]^{T}\right\} .
\end{aligned}
$$

Based on Equations (25)-(34), (39), and (54), we have

$$
\begin{aligned}
& L V\left(t, x_{t}\right)+\alpha V\left(t, x_{t}\right)-\frac{\alpha}{\omega_{m}^{2}} \omega^{T}(t) \omega(t) \leq \xi_{2}^{T}(t)\left(\widehat{\Psi}_{i}+\Pi_{2}\right) \xi_{2}(t) \\
& \quad+\eta^{T}(t)\left(\sum_{j \in U_{u k}^{i}} \lambda_{i j}\left(P_{j}-W_{i}\right)\right) \eta(t),
\end{aligned}
$$

where $\widehat{\Psi}_{i}, \Pi_{2}$ are the same as defined in Theorem 1. Using the $S$-procedure in [4], one can see that this condition is implied by the existence of a non-negative scalar $\varepsilon>0$ such that

$$
\begin{aligned}
& L V\left(t, x_{t}\right)+\alpha V\left(t, x_{t}\right)-\frac{\alpha}{\omega_{m}^{2}} \omega^{T}(t) \omega(t) \leq \xi_{2}^{T}(t)\left(\widehat{\Psi}_{i}+\Pi_{2}\right) \xi_{2}(t)+\eta^{T}(t)\left(\sum_{j \in U_{u k}^{i}} \lambda_{i j}\left(P_{j}-W_{i}\right)\right) \eta(t) \\
& \quad+\varepsilon\left\{\left[E_{i a} x(t)+E_{i b} x(t-h(t))+E_{i d} \omega(t)\right]^{T}\left[E_{i a} x(t)+E_{i b} x(t-h(t))+E_{i d} \omega(t)\right]-u^{T} u\right\}<0,
\end{aligned}
$$

for all $\xi_{2}(t) \neq 0$. By using Lemma 5 , the matrix inequalities (22)-(24) imply (56).

It should be noted that $\Psi_{i}+\Pi_{1}<0, \quad \widehat{\Psi}_{i}+\Pi_{2}<0$ according to inequalities (22)-(24), which implies that $L V\left(t, x_{t}\right)+\alpha V\left(t, x_{t}\right)-\left(\alpha / \omega_{m}^{2}\right) \omega^{T}(t) \omega(t)<0$.

In conclusion, $\quad L V\left(t, x_{t}\right)+\alpha V\left(t, x_{t}\right)-\left(\alpha / \omega_{m}^{2}\right)$ $\omega^{T}(t) \omega(t)<0$ is true on the basis of equations (21)-(24). Since $P_{i}=\left[p_{i, j}\right]_{5 \times 5}$, there is a positive matrix $\widetilde{P}_{1, i} \in \mathbb{R}^{n \times n}$ such that

$$
P_{i} \geq\left[\begin{array}{ccccc}
\widetilde{P}_{1, i} & 0 & 0 & 0 & 0 \\
0 & 0 & 0 & 0 & 0 \\
0 & 0 & 0 & 0 & 0 \\
0 & 0 & 0 & 0 & 0 \\
0 & 0 & 0 & 0 & 0
\end{array}\right] .
$$

We further have the inequality $x^{T}(t) \widetilde{P}_{1, i} x(t) \leq$ $V_{1}\left(t, x_{t}\right) \leq V\left(t, x_{t}\right) \leq 1 \quad$ when $h(t) \in\left[h_{1}, h_{2}\right]$. Hence,
$V\left(t, x_{t}\right) \leq 1$ is true by using Lemma 1 . This completes the proof.

Remark 1. We choose an augmented Lyapunov-Krasovskii functional $V_{1}\left(t, x_{t}\right)=\eta^{T}(t) P_{r_{t}} \eta(t)$ to establish more general delay-dependent conditions, where $P_{r_{t}}$ is a fifth-order matrix and $\eta(t)$ is a five-dimensional column vector. Therefore, the reachable set estimation criteria can utilize more information on state variables via using these augmented variables in the Lyapunov-Krasovskii functional.

Remark 2. Parameter $\gamma$ is used to divide the time-delay interval into two subintervals in this paper. $h(t)$ is the timevarying delay satisfying $0 \leq h_{1} \leq h(t) \leq h_{2}$. Generally, the authors divide the time-delay interval into two or more equal subintervals in previous studies to get less conservative stability criteria. Different from them, the time-delay interval $\left[h_{1}, h_{2}\right]$ in our study is partitioned into $\left[h_{1}, h_{m}\right]$ and $\left[h_{m}, h_{2}\right]$ by introducing an adjustable parameter $\gamma$, where $h_{m}=\gamma h_{1}+(1-\gamma) h_{2}, 0 \leq \gamma \leq 1$; when $\gamma=0.5$, the two 
subintervals are equal as those in the literatures. Moreover, the integral interval is decomposed in the same way to estimate the bounds of integral terms more exactly.

Remark 3. An optimized integral inequality is provided to deal with the integral term $-\left(h_{m}-h_{1}\right) \int_{t-h_{m}}^{t-h_{1}} \dot{x}^{T}(s) R_{5} \dot{x}(s) \mathrm{d} s$ and $-\left(h_{2}-h_{m}\right) \int_{t-h}^{t-h_{m}} \dot{x}^{T}(s) R_{6} \dot{x}(s) \mathrm{d} s$. Recently, the reciprocally convex combination approach $[18,25,27]$ has been widely used to deduce the results. According to the applications shown in these literatures, it is easy to see that the results based on this method are less conservative than the existing ones. Therefore, we adopt the distinguished Wirtinger integral inequality [23] together with the reciprocally convex combination inequality to handle these two integral terms to get more general reachable set estimation criteria.

Corollary 1. Consider the uncertain Markov jump system (1)-(4) with all elements completely known in transition rate matrix (5); if there exist real matrices $P_{i}=\left[\begin{array}{ccccc}P_{i 11} & P_{i 12} & P_{i 13} & P_{i 14} & P_{i 15} \\ * & P_{i 22} & P_{i 23} & P_{i 24} & P_{i 25} \\ * & * & P_{i 33} & P_{i 34} & P_{i 35} \\ * & * & * & P_{i 44} & P_{i 45} \\ * & * & * & * & P_{i 55}\end{array}\right]>0, \quad W_{i}>0(i=1,2, \ldots$,

N), $\widetilde{P}_{1, i}>0, Q_{1}>0, Q_{2}>0, Q_{3}>0$ and $R_{i}>0(i=1, \ldots, 6)$, $\widetilde{R}_{5}=\left[\begin{array}{ccc}R_{5} & 0 & 0 \\ 0 & 3 R_{5} & 0 \\ 0 & 0 & 5 R_{5}\end{array}\right], \quad \widetilde{R}_{6}=\left[\begin{array}{ccc}R_{6} & 0 & 0 \\ 0 & 3 R_{6} & 0 \\ 0 & 0 & 5 R_{6}\end{array}\right]$, any matrices $M_{i}(i=1,2), X=\left[\begin{array}{lll}X_{11} & X_{12} & X_{13} \\ X_{21} & X_{22} & X_{23} \\ X_{31} & X_{32} & X_{33}\end{array}\right], \quad Y=\left[\begin{array}{lll}Y_{11} & Y_{12} & Y_{13} \\ Y_{21} & Y_{22} & Y_{23} \\ Y_{31} & Y_{32} & Y_{33}\end{array}\right]$ with appropriate dimension such that $\left[\begin{array}{cc}\widetilde{R}_{5} & X \\ * & \widetilde{R}_{5}\end{array}\right]>0$, $\left[\begin{array}{cc}\widetilde{R}_{6} & Y \\ * & \widetilde{R}_{6}\end{array}\right]>0, P_{i} \geq\left[\begin{array}{ccccc}\widetilde{P}_{1, i} & 0 & 0 & 0 & 0 \\ * & 0 & 0 & 0 & 0 \\ * & * & 0 & 0 & 0 \\ * & * & * & 0 & 0 \\ * & * & * & 0 & 0\end{array}\right]$ a scalar $\varepsilon>0$ such

that the LMIs equations (21)-(22) hold, which all elements $\Psi_{i}, \widehat{\Psi}_{i}, \Phi_{1}, \Phi_{2}, \Phi_{3}, \Pi_{1}$, and $\Pi_{2}$ are defined in Appendix $B$.

Corollary 2. Consider the uncertain Markov jump system (1)-(4) with all elements completely unknown in transition rate matrix (5); if there exist real matrices $P_{i}=\left[\begin{array}{ccccc}P_{i 11} & P_{i 12} & P_{i 13} & P_{i 14} & P_{i 15} \\ * & P_{i 22} & P_{i 23} & P_{i 24} & P_{i 25} \\ * & * & P_{i 33} & P_{i 34} & P_{i 35} \\ * & * & * & P_{i 44} & P_{i 45} \\ * & * & * & * & P_{i 55}\end{array}\right]>0, \quad W_{i}>0(i=1,2, \ldots$,

N), $\widetilde{P}_{1, i}>0, \quad Q_{1}>0, \quad Q_{2}>0, \quad Q_{3}>0, \quad R_{i}>0(i=1, \ldots, 6)$, $\widetilde{R}_{5}=\left[\begin{array}{ccc}R_{5} & 0 & 0 \\ 0 & 3 R_{5} & 0 \\ 0 & 0 & 5 R_{5}\end{array}\right], \widetilde{R}_{6}=\left[\begin{array}{ccc}R_{6} & 0 & 0 \\ 0 & 3 R_{6} & 0 \\ 0 & 0 & 5 R_{6}\end{array}\right]$, any matrices $M_{i}(i=1,2), X=\left[\begin{array}{lll}X_{11} & X_{12} & X_{13} \\ X_{21} & X_{22} & X_{23} \\ X_{31} & X_{32} & X_{33}\end{array}\right], \quad Y=\left[\begin{array}{lll}Y_{11} & Y_{12} & Y_{13} \\ Y_{21} & Y_{22} & Y_{23} \\ Y_{31} & Y_{32} & Y_{33}\end{array}\right]$ with appropriate dimension such that $\left[\begin{array}{cc}\widetilde{R}_{5} & X \\ * & \widetilde{R}_{5}\end{array}\right]>0$, $\left[\begin{array}{cc}\widetilde{R}_{6} & Y \\ * & \widetilde{R}_{6}\end{array}\right]>0, P_{i} \geq\left[\begin{array}{ccccc}\widetilde{P}_{1, i} & 0 & 0 & 0 & 0 \\ * & 0 & 0 & 0 & 0 \\ * & * & 0 & 0 & 0 \\ * & * & * & 0 & 0 \\ * & * & * & 0 & 0\end{array}\right]$, scalar $\varepsilon>0$ such that the LMIs equations (21) and (22) hold, where $\Psi_{i}, \widehat{\Psi}_{i}, \Phi_{1}, \Phi_{2}$, $\Phi_{3}, \Pi_{1}$, and $\Pi_{2}$ are defined in Appendix $C$.

Next, we consider the following Markov jump system with disturbances

$$
\left\{\begin{array}{l}
\dot{x}(t)=A_{\left(t, r_{t}\right)} x(t)+B_{\left(t, r_{t}\right)} x(t-h(t))+D_{\left(t, r_{t}\right)} \omega(t), \\
x(t) \equiv 0, \quad \forall t \in\left[-h_{2}, 0\right] .
\end{array}\right.
$$

Theorem 2 is a result for the ellipsoidal bound of a reachable set for a Markov jump time-delayed system (58) having constraints in equations (2) and (3).

Theorem 2. Consider the Markov jump system (56) with all elements partly known in transition rate matrix (5); if there exist real matrices $Q_{1}>0, \quad Q_{2}>0, \quad Q_{3}>0$, $P_{i}=\left[\begin{array}{cccc}P_{i 11} & P_{i 12} & P_{i 13} & P_{i 14} \\ * & P_{i 22} & P_{i 23} & P_{i 24} \\ * & * & P_{i 33} & P_{i 34} \\ * & * & * & P_{i 44}\end{array}\right]>0, \quad W_{i}>0, \quad \widetilde{P}_{1,1}>0$ and $R_{i}>0(i=1, \ldots, 6)$,

$$
\widetilde{R}_{5}=\left[\begin{array}{ccc}
R_{5} & 0 & 0 \\
0 & 3 R_{5} & 0 \\
0 & 0 & 5 R_{5}
\end{array}\right]
$$

$\widetilde{R}_{6}=\left[\begin{array}{ccc}R_{6} & 0 & 0 \\ 0 & 3 R_{6} & 0 \\ 0 & 0 & 5 R_{6}\end{array}\right]$, any matrices $M_{i}(i=1,2)$, $X=\left[\begin{array}{lll}X_{11} & X_{12} & X_{13} \\ X_{21} & X_{22} & X_{23} \\ X_{31} & X_{32} & X_{33}\end{array}\right], Y=\left[\begin{array}{lll}Y_{11} & Y_{12} & Y_{13} \\ Y_{21} & Y_{22} & Y_{23} \\ Y_{31} & Y_{32} & Y_{33}\end{array}\right]$ with appropriate dimension such that $\left[\begin{array}{cc}\widetilde{R}_{5} & X \\ * & \widetilde{R}_{5}\end{array}\right]>0,\left[\begin{array}{cc}\widetilde{R}_{6} & Y \\ * & \widetilde{R}_{6}\end{array}\right]>0$, $P_{i} \geq\left[\begin{array}{cccc}\widetilde{P}_{1,1} & 0 & 0 & 0 \\ * & 0 & 0 & 0 \\ * & * & 0 & 0 \\ * & * & * & 0\end{array}\right]$, satisfying the following matrix inequalities:

$$
\begin{aligned}
& \Psi_{i}+\Phi_{1}<0, \\
& \widehat{\Psi}_{i}+\Phi_{2}<0, \\
& \widehat{\Psi}_{i}+\Phi_{3}<0, \\
& \Psi_{i}+\Pi_{1}<0, \\
& \widehat{\Psi}_{i}+\Pi_{2}<0, \\
& P_{j}-W_{i} \leq 0, \quad j \in U_{u k}^{i}, j \neq i, \\
& P_{j}-W_{i} \geq 0, \quad j \in U_{u k}^{i}, j=i,
\end{aligned}
$$

where all the elements are defined in Appendix D. 
Then, the reachable sets of system (58) having the constraints (2) and (3) are bounded by a boundary $\mathfrak{\Im}(P)$ defined in equation (13).

Proof. of Theorem 2 Following a similar line as in the proof of Theorem 1, one can simply obtain this theorem. This completes our proof.

Corollary 3. Consider the Markov jump system (58) with all elements completely known in transition rate matrix (5); if there exist real matrices $P_{i}=\left[\begin{array}{cccc}P_{i 11} & P_{i 12} & P_{i 13} & P_{i 14} \\ * & P_{i 22} & P_{i 23} & P_{i 24} \\ * & * & P_{i 33} & P_{i 34} \\ * & * & * & P_{i 44}\end{array}\right]>0$, $\widetilde{P}_{1,1}>0, Q_{1}>0, Q_{2}>0, Q_{3}>0$ and $R_{i}>0(i=1, \ldots, 6)$, $\widetilde{R}_{5}=\left[\begin{array}{ccc}R_{5} & 0 & 0 \\ 0 & 3 R_{5} & 0 \\ 0 & 0 & 5 R_{5}\end{array}\right], \quad \widetilde{R}_{6}=\left[\begin{array}{ccc}R_{6} & 0 & 0 \\ 0 & 3 R_{6} & 0 \\ 0 & 0 & 5 R_{6}\end{array}\right]$, any matrices $M_{i}(i=1,2), \quad X=\left[\begin{array}{lll}X_{11} & X_{12} & X_{13} \\ X_{21} & X_{22} & X_{23} \\ X_{31} & X_{32} & X_{33}\end{array}\right], \quad Y=\left[\begin{array}{lll}Y_{11} & Y_{12} & Y_{13} \\ Y_{21} & Y_{22} & Y_{23} \\ Y_{31} & Y_{32} & Y_{33}\end{array}\right]$ with appropriate dimension such that $\left[\begin{array}{cc}\widetilde{R}_{5} & X \\ * & \widetilde{R}_{5}\end{array}\right]>0$, $\left[\begin{array}{cc}\widetilde{R}_{6} & Y \\ * & \widetilde{R}_{6}\end{array}\right]>0, P_{i} \geq\left[\begin{array}{cccc}\widetilde{P}_{1,1} & 0 & 0 & 0 \\ * & 0 & 0 & 0 \\ * & * & 0 & 0 \\ * & * & * & 0\end{array}\right]$, satisfying the following matrix inequalities:

$$
\begin{gathered}
\Psi_{i}+\Phi_{1}<0, \\
\widehat{\Psi}_{i}+\Phi_{2}<0, \\
\widehat{\Psi}_{i}+\Phi_{3}<0, \\
\Psi_{i}+\Pi_{1}<0, \\
\widehat{\Psi}_{i}+\Pi_{2}<0,
\end{gathered}
$$

where all elements are defined in Appendix E.

Corollary 4. Consider the Markov jump system (58) with all elements completely unknown in transition rate matrix (5); if there exist real matrices $P_{i}=\left[\begin{array}{cccc}P_{i 11} & P_{i 12} & P_{i 13} & P_{i 14} \\ * & P_{i 22} & P_{i 23} & P_{i 24} \\ * & * & P_{i 33} & P_{i 34} \\ * & * & * & P_{i 44}\end{array}\right]>0$, $\widetilde{P}_{1,1}>0, Q_{1}>0, Q_{2}>0, Q_{3}>0$ and $R_{i}>0(i=1, \ldots, 6)$, $\widetilde{R}_{5}=\left[\begin{array}{ccc}R_{5} & 0 & 0 \\ 0 & 3 R_{5} & 0 \\ 0 & 0 & 5 R_{5}\end{array}\right], \quad \widetilde{R}_{6}=\left[\begin{array}{ccc}R_{6} & 0 & 0 \\ 0 & 3 R_{6} & 0 \\ 0 & 0 & 5 R_{6}\end{array}\right]$, any matrices $M_{i}(i=1,2), \quad X=\left[\begin{array}{lll}X_{11} & X_{12} & X_{13} \\ X_{21} & X_{22} & X_{23} \\ X_{31} & X_{32} & X_{33}\end{array}\right], \quad Y=\left[\begin{array}{lll}Y_{11} & Y_{12} & Y_{13} \\ Y_{21} & Y_{22} & Y_{23} \\ Y_{31} & Y_{32} & Y_{33}\end{array}\right]$ with appropriate dimension such that $\left[\begin{array}{cc}\widetilde{R}_{5} & X \\ * & \widetilde{R}_{5}\end{array}\right]>0$, $\left[\begin{array}{cc}\widetilde{R}_{6} & Y \\ * & \widetilde{R}_{6}\end{array}\right]>0, P_{i} \geq\left[\begin{array}{cccc}\widetilde{P}_{1,1} & 0 & 0 & 0 \\ * & 0 & 0 & 0 \\ * & * & 0 & 0 \\ * & * & * & 0\end{array}\right]$, satisfying the following matrix inequalities:

$$
\begin{aligned}
& \Psi_{i}+\Phi_{1}<0, \\
& \widehat{\Psi}_{i}+\Phi_{2}<0, \\
& \widehat{\Psi}_{i}+\Phi_{3}<0, \\
& \Psi_{i}+\Pi_{1}<0, \\
& \widehat{\Psi}_{i}+\Pi_{2}<0,
\end{aligned}
$$

where all elements are defined in Appendix F.

Remark 4. Since this is an estimation problem, our aim is to find an ellipsoid as small as possible to bound the reachable sets of system (1).We use a simple approximation as that in [7]. That is, maximize $\delta>0$ subject to $\widetilde{P}_{1,1} \geq \delta I$, which can be transformed into the following optimization problem:

$$
\begin{aligned}
& \min \bar{\delta}=\frac{1}{\delta} \\
& \text { s.t. } \begin{cases}i) & {\left[\begin{array}{cc}
\bar{\delta} I & I \\
I & \widetilde{P}_{1,1}
\end{array}\right] \geq 0,} \\
\text { ii) } & \text { Eqs. }(13)-(16) \text { or }(48)-(51),\end{cases}
\end{aligned}
$$

\section{Numerical Examples}

In this section, two examples are used to demonstrate the effectiveness and correctness of the main results derived above.

Example 1. Consider the following uncertain time-delayed system (58) which has been studied in $[9,11]$ :

$$
\begin{aligned}
A_{1} & =\left[\begin{array}{cc}
-2 & 0 \\
0 & -0.7
\end{array}\right], \\
B_{1} & =\left[\begin{array}{cc}
-1 & 0 \\
-1 & -0.9
\end{array}\right], \\
D_{1} & =\left[\begin{array}{cc}
-0.5 \\
1
\end{array}\right], \\
A_{2} & =\left[\begin{array}{cc}
-2 & 0 \\
0 & -1.1
\end{array}\right], \\
B_{2} & =\left[\begin{array}{cc}
-1 & 0 \\
-1 & -1.1
\end{array}\right], \\
D_{2} & =D_{1}, \omega^{T}(t) \omega(t) \leq \omega_{m}^{2}=1, h_{1}=0 .
\end{aligned}
$$

The transition rate matrix $\Lambda$ is considered as in the following three cases.

Case 1. The transition rate matrix $\Lambda$ is completely known, which is considered as $\Lambda=\left[\begin{array}{cc}-0.6 & 0.6 \\ 0.2 & -0.2\end{array}\right]$.

Case 2. The transition rate matrix $\Lambda$ is partly known, which is considered as $\Lambda=\left[\begin{array}{cc}-0.6 & 0.6 \\ ? & ?\end{array}\right]$. 
Case 3. The transition rate matrix $\Lambda$ is completely unknown, which is considered as $\Lambda=\left[\begin{array}{ll}? & ? \\ ? & ?\end{array}\right]$.

By solving the optimization problem (59)-(62), the allowable minimum values of $\bar{\delta}$ obtained by different methods for $0 \leq h(t) \leq 0.7$ and $0 \leq h(t) \leq 0.75$ are listed in Table 1 . According the Table 1, it is inescapably clear that our results decrease the size of the ellipsoid significantly.

Furthermore, for the parameters listed above, let $h_{2}=0.75, \alpha=0.7$, and we can get the following feasible solutions by Theorem 2 in this paper. In this example, when the transition probability matrix is completely unknown (at this point, the state trajectory is general switching system, not the system studied in this paper), it is a general switching system, such as $[9,11]$. But when the $\Lambda$ is partly unknown or completely known, it is the Markov jump system. And there are two elliptical boundaries in the picture. So, we choose the smaller one as the reachable set boundary. Due to the limitation of the length of this paper, we just show some of them here, and the reachable set is $\cap_{i=1}^{2} \mathfrak{\Im}\left(\widetilde{P}_{1, i}\right)$.

By using Theorem 2 and solving the problem (59)-(62) in Case 2, we can obtain

$$
\begin{aligned}
P_{i 11} & =\left[\begin{array}{cc}
2.4425 & -0.4772 \\
-0.4772 & 1.0098
\end{array}\right], \\
P_{i 44} & =\left[\begin{array}{cc}
24.8867 & 0.3433 \\
0.3433 & 24.9046
\end{array}\right], \\
Q_{3} & =\left[\begin{array}{cc}
175.9261 & 0.4467 \\
0.4467 & 173.8194
\end{array}\right], \\
R_{6} & =\left[\begin{array}{cc}
0.0493 & -0.0035 \\
-0.0035 & 0.0365
\end{array}\right], \\
X_{11} & =\left[\begin{array}{cc}
-50.2037 & 0.0891 \\
0.0893 & -50.3448
\end{array}\right], \\
Y_{11} & =\left[\begin{array}{cc}
-52.9810 & 0.2084 \\
-0.2832 & -52.6961
\end{array}\right], \\
\widetilde{P}_{1,1} & =\left[\begin{array}{cc}
1.6344 & -0.3169 \\
-0.3169 & 0.6826
\end{array}\right] .
\end{aligned}
$$

By using Corollary 3 and solving the problems (63) and (64) in Case 1, we can obtain

$$
\begin{aligned}
P_{i 11} & =\left[\begin{array}{cc}
4.3786 & -0.5368 \\
-0.5368 & 2.1556
\end{array}\right], \\
P_{i 44} & =\left[\begin{array}{ll}
72.9859 & 0.3683 \\
0.3683 & 72.5227
\end{array}\right], \\
Q_{3} & =\left[\begin{array}{ll}
474.5381 & 0.7949 \\
0.7949 & 467.5645
\end{array}\right], \\
\widetilde{P}_{1,1} & =\left[\begin{array}{ll}
2.9222 & -0.3632 \\
-0.3632 & 1.4403
\end{array}\right] .
\end{aligned}
$$

By using Corollary 4 and solving the problems (65) and (66) in Case 3, we can obtain

$$
\begin{aligned}
P_{i 11} & =\left[\begin{array}{cc}
4.9321 & -0.4496 \\
-0.4496 & 2.5786
\end{array}\right], \\
P_{i 44} & =\left[\begin{array}{cc}
99.7440 & 0.3944 \\
0.3944 & 99.3030
\end{array}\right], \\
Q_{3} & =\left[\begin{array}{cc}
678.1573 & 1.0381 \\
1.0381 & 672.4074
\end{array}\right], \\
R_{3} & =\left[\begin{array}{cc}
0.1860 & -0.0122 \\
-0.0122 & 0.1374
\end{array}\right], \\
X_{11} & =\left[\begin{array}{cc}
-195.7636 & 0.4919 \\
0.4548 & -197.1652
\end{array}\right], \\
Y_{11} & =\left[\begin{array}{cc}
-203.4827 & 0.7165 \\
-0.8392 & -202.9476
\end{array}\right], \\
\widetilde{P}_{1,1} & =\left[\begin{array}{cc}
3.2976 & -0.3031 \\
-0.3031 & 1.7280
\end{array}\right] .
\end{aligned}
$$


TABLE 1: The minimum sizes of ellipsoidal bound $(\bar{\delta})$ obtained by different methods.

\begin{tabular}{lccccccccc}
\hline Method & {$[28]$} & {$[22]$} & {$[29]$} & {$[30]$} & {$[13]$} & {$[12]$} & {$[11]$ cor 3.3 } & [11] cor 3.4 & Corollary 4 \\
\hline $0 \leq h(t) \leq 0.7$ & 2.97 & 1.9151 & 1.89 & 1.79 & 1.38 & 1.15 & 1.1096 & 1.1375 & 0.9279 \\
$0 \leq h(t) \leq 0.75$ & 3.34 & 2.3199 & 2.28 & 2.03 & 1.27 & 1.34 & 1.1551 & 1.2064 & 0.5982 \\
\hline
\end{tabular}

Figure 1 is the plot of the state trajectory of system (56). Figures $2-4$ are the plots of the ellipsoidal sets $\mathfrak{I}$ defined in equation (13), which are obtained in Theorem 2 and Corollaries 3 and 4 when $h_{2}=0.75$, respectively.

Example 2. Consider the uncertain MJSs with time-varying delays and disturbances:

$$
\begin{aligned}
\dot{x}(t)= & \left(A_{\left(t, r_{t}\right)}+\Delta A_{\left(t, r_{t}\right)}(t)\right) x(t) \\
& +\left(B_{\left(t, r_{t}\right)}+\Delta B_{\left(t, r_{t}\right)}(t)\right) x(t-h(t)) \\
& +\left(D_{\left(t, r_{t}\right)}+\Delta D_{\left(t, r_{t}\right)}(t)\right) \omega(t),
\end{aligned}
$$

where

$$
\begin{aligned}
& A_{1}=\left[\begin{array}{cc}
-5 & 0 \\
0 & -6
\end{array}\right], \\
& B_{1}=\left[\begin{array}{cc}
-1.6 & 0 \\
-1.8 & -1.5
\end{array}\right], \\
& D_{1}=\left[\begin{array}{c}
0.5 \\
-1
\end{array}\right], \\
& L_{1}=\left[\begin{array}{cc}
0.1 & 0 \\
0 & 0.1
\end{array}\right], \\
& E_{13}=0, \\
& A_{2}=\left[\begin{array}{cc}
-4 & 0 \\
0 & -5
\end{array}\right], \\
& B_{2}=\left[\begin{array}{cc}
-2 & 0 \\
-0.9 & -1.2
\end{array}\right], \\
& D_{2}=\left[\begin{array}{cc}
-0.5 & 1
\end{array}\right], \\
& L_{2}=\left[\begin{array}{cc}
0 & 0 \\
0 & -0.1
\end{array}\right], \\
& E_{23}=0, \\
& E_{11}=\left[\begin{array}{cc}
0 & 0 \\
0 & -0.1
\end{array}\right], \\
& E_{12}=\left[\begin{array}{cc}
-0.1 & 0 \\
0 & 0.1
\end{array}\right], \\
& E_{21}=\left[\begin{array}{cc}
0 & 0 \\
0 & 0.1
\end{array}\right], \\
& E_{22}=\left[\begin{array}{cc}
0.1 & 0 \\
0 & 0.1
\end{array}\right],
\end{aligned}
$$
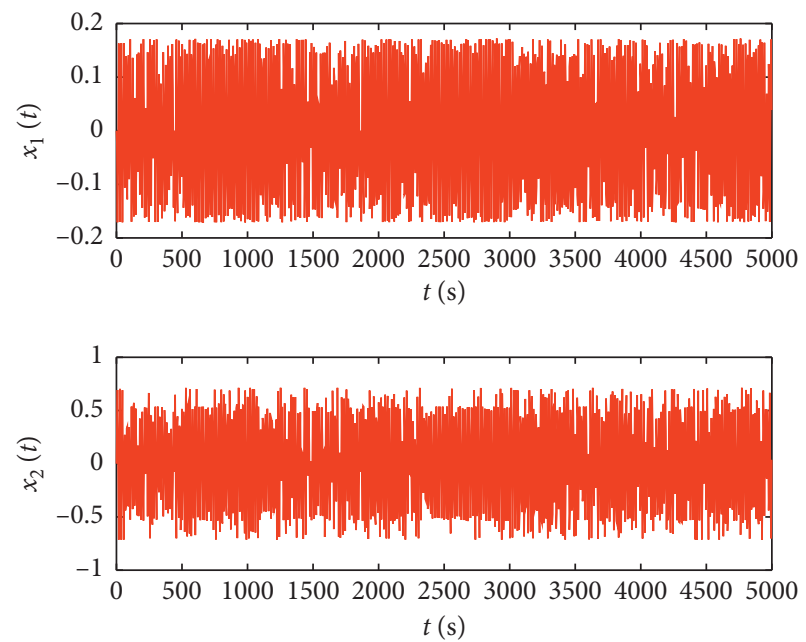

FIGURE 1: The time responses of state variable $x(t)$ of Markov jump system (1) for Case 1.

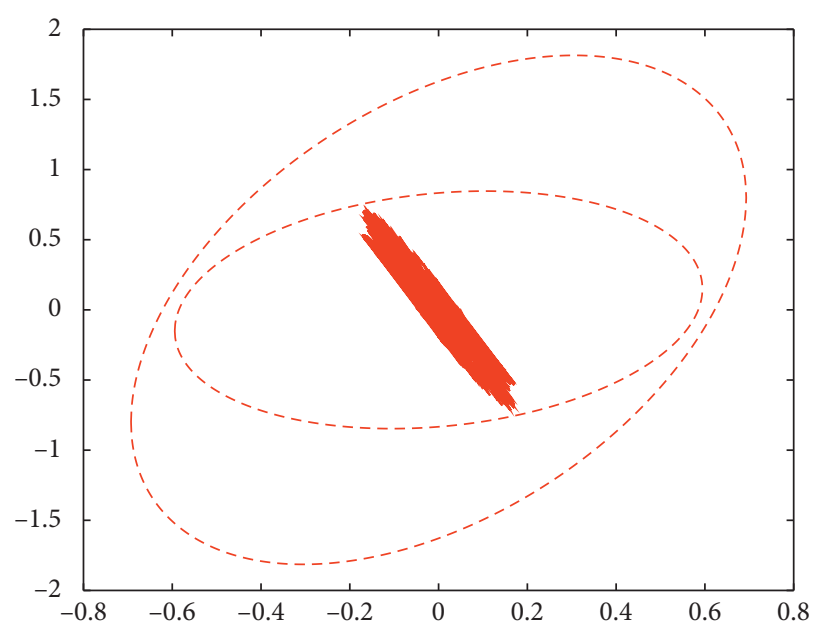

Figure 2: The ellipsoidal bound $\mathfrak{I}$ and state trajectory of system (58) for Case 1.

By using Corollary 1 and solving the optimization problem (72) in Case 1, we can get that the minimization of $\bar{\delta}$ is 0.0785 (the short half axis length of the ellipsoid is 0.2802 ) when $\alpha=0.7$ and the corresponding feasible matrices are given

$\widetilde{P}_{1,1}=\left[\begin{array}{cc}21.3278 & -0.3828 \\ -0.3828 & 12.7572\end{array}\right], \widetilde{P}_{1,2}=\left[\begin{array}{cc}10.2938 & 0.9042 \\ 0.9042 & 10.2938\end{array}\right]$.

The reachable sets of system (72) in Case 1 are bounded by a intersection of two ellipsoids: $\cap_{i=1}^{2} \mathfrak{T}\left(\widetilde{P}_{1, i}\right)$, which is depicted in Figure 5.

By using Theorem 1 and solving the optimization problem (72) in Case 2, we can get that the minimization of $\bar{\delta}$ 


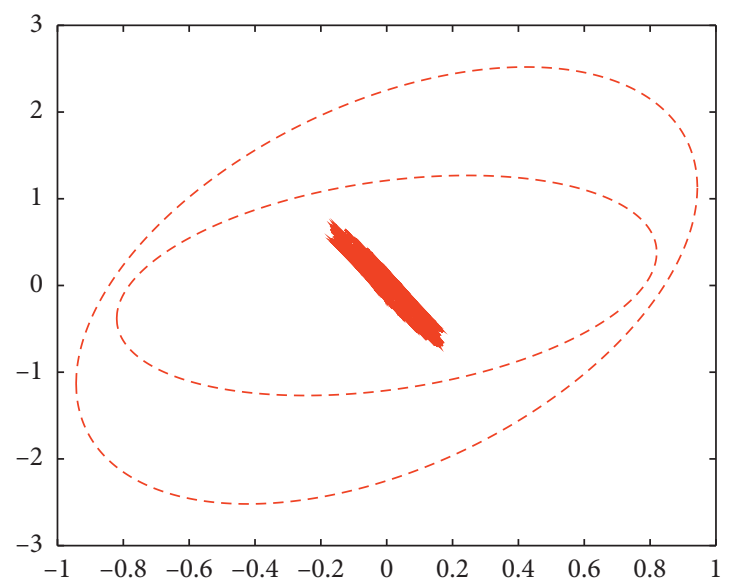

Figure 3: The ellipsoidal bound $\mathfrak{\Im}$ and state trajectory of system (58) for Case 2.

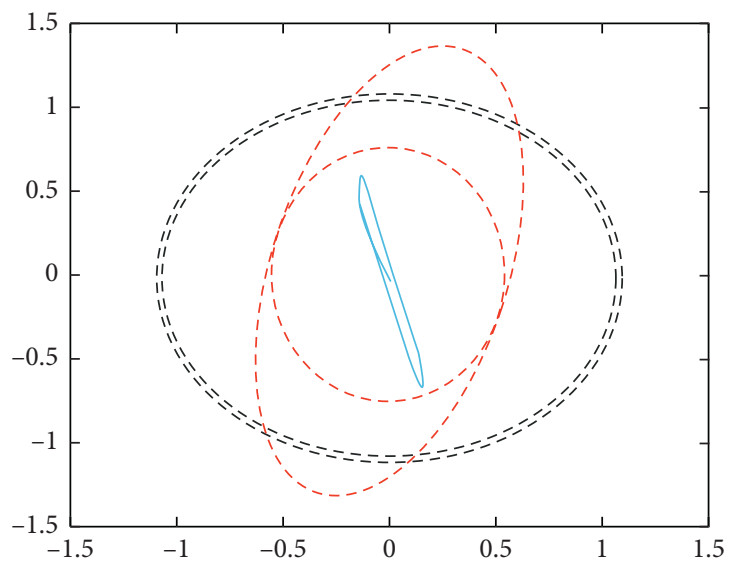

FIgURE 4: The ellipsoidal bound $\mathfrak{I}$ and state trajectory of system (58) for Case 3.

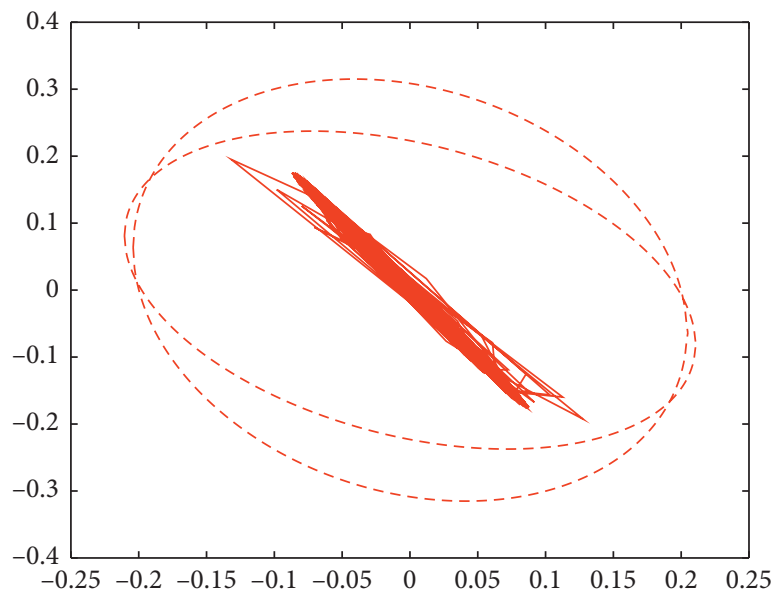

Figure 5: The ellipsoidal bound $\mathfrak{I}$ and state trajectory of system(72) for Case 1. 


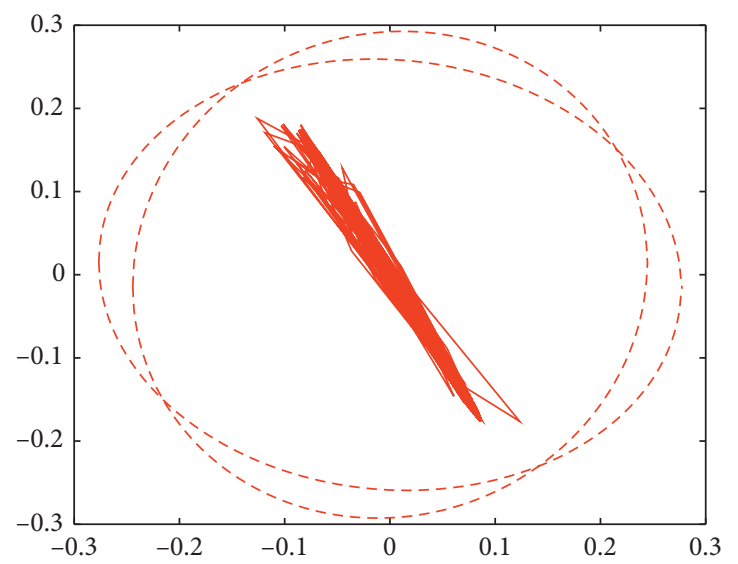

Figure 6: The ellipsoidal bound $\mathfrak{I}$ and state trajectory of system (72) for Case 2.

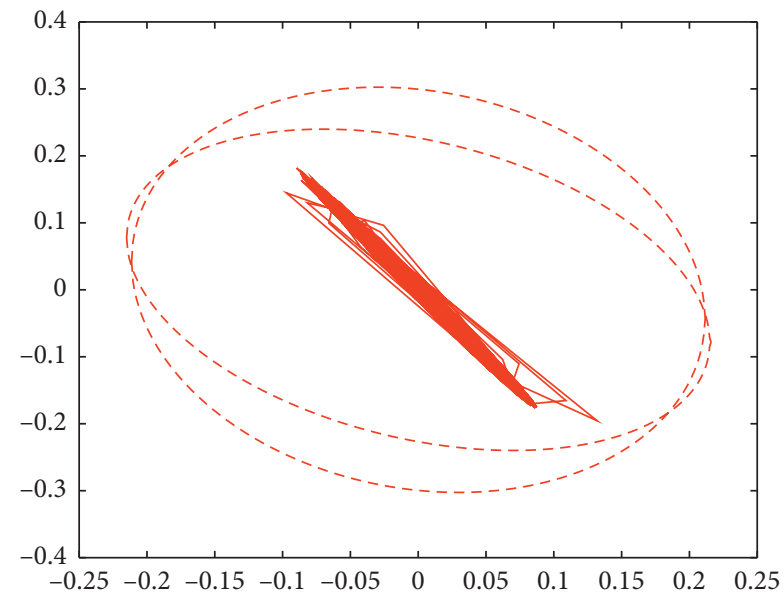

FIgURE 7: The ellipsoidal bound $\mathfrak{I}$ and state trajectory of system (72) for Case 3.

is 0.0524 (the short half axis length of the ellipsoid is 0.2288 ) when $\alpha=0.7$ and the corresponding feasible matrices are given $\quad$ as $\quad \widetilde{P}_{1,1}=\left[\begin{array}{cc}32.8001 & 2.0190 \\ 2.0190 & 19.3954\end{array}\right], \widetilde{P}_{1,2}=$ $\left[\begin{array}{cc}15.8731 & 2.5565 \\ 2.5565 & 13.5311\end{array}\right]$. The reachable sets of system (72) in Case 1 are bounded by a intersection of two ellipsoids: $\cap_{i=1}^{2} \mathfrak{J}\left(\widetilde{P}_{1, i}\right)$, which is depicted in Figure 6.

By using Corollary 2 and solving the optimization problem (72) in Case 3, we can get that the minimization of $\bar{\delta}$ is 0.0598 (the short half axis length of the ellipsoid is 0.2445 ) when $\alpha=0.7$ and the corresponding feasible matrices are given $\quad$ as $\quad \widetilde{P}_{1,1}=\left[\begin{array}{cc}32.8028 & 4.4496 \\ 4.4496 & 17.9617\end{array}\right], \widetilde{P}_{1,2}=$ $\left[\begin{array}{cc}21.1376 & 5.7135 \\ 5.7135 & 14.6146\end{array}\right]$. The reachable sets of system (72) in Case 1 are bounded by a intersection of two ellipsoids: $\cap_{i=1}^{2} \mathfrak{I}\left(\widetilde{P}_{1, i}\right)$, which is depicted in Figure 7 .
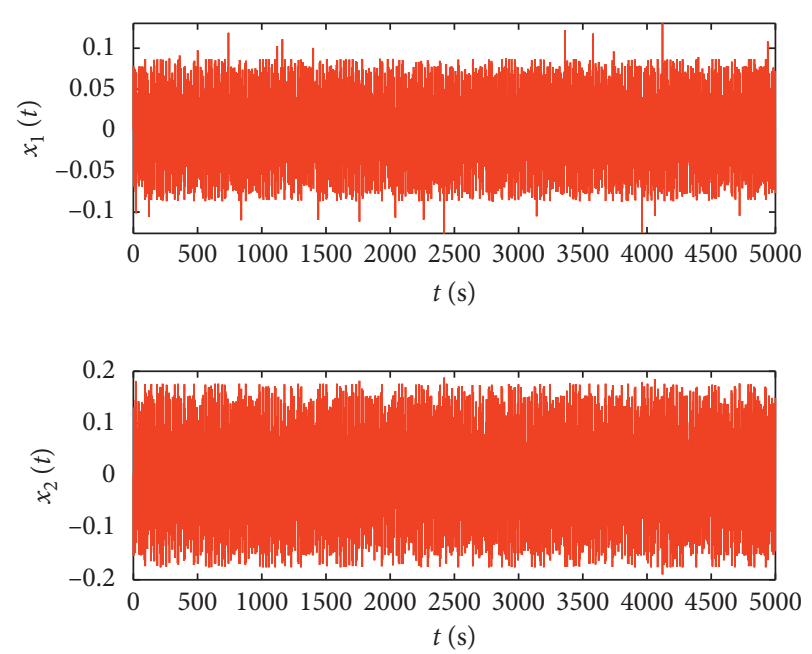

FIgURE 8: The time responses of state variable $x(t)$ of Markov jump system (72) for Case 1 .

Figure 8 is the plot of the time responses of state variable for system (72). Figures 5-7 are the plots of the ellipsoidal sets $\mathfrak{I}$ defined in equation (13), which are obtained in Theorem 1 and Corollaries 1 and 2 when $h_{2}=0.75$, respectively.

\section{Conclusion}

The reachable set bounding for uncertain Markov jump systems with time-varying delays and disturbances has been investigated in our study. We partition the time-varying delay into two nonuniform subintervals and consider the delay in these two cases separately. Furthermore, some new reachable set estimation conditions are derived in terms of linear matrix inequalities by constructing a novel augmented Lyapunov-Krasovskii functional, combining with optimized integral inequality which is based on distinguished Wirtinger integral inequality and reciprocally convex combination inequality. Finally, the feasibility and the 
comparisons with recent results obtained in the latest literatures are shown through numerical examples.

\section{Appendix}

A. The Representation of $\Psi_{i}, \widehat{\Psi}_{i}, \Phi_{1}$, $\Phi_{2}, \Phi_{3}, \Pi_{1}, \Pi_{2}$ in Theorem 1

$$
\Psi_{i}=\left[\begin{array}{cccc}
\Psi_{i 11} & \Psi_{i 12} & \Psi_{i 13} & \Psi_{i 14} \\
* & \Psi_{i 22} & \Psi_{i 23} & \Psi_{i 24} \\
* & * & \varphi_{i 17,17} & 0 \\
* & * & * & -\varepsilon I
\end{array}\right],
$$

$$
\Psi_{i 11}=\left[\begin{array}{ccccccccc}
\varphi_{i 11} & \varphi_{i 12} & \varphi_{i 13} & \varphi_{i 14} & \varphi_{i 15} & \varphi_{i 16} & \varphi_{i 17} & \varphi_{i 18} & \varphi_{i 19} \\
* & \varphi_{22} & 0 & \varphi_{i 24} & 0 & 0 & \varphi_{i 27} & \varphi_{i 28} & \varphi_{i 29} \\
* & * & \varphi_{33} & 0 & 0 & 0 & \varphi_{i 37} & \varphi_{i 38} & \varphi_{i 39} \\
* & * & * & 0 & 0 & 0 & \varphi_{i 47} & \varphi_{i 48} & \varphi_{i 49} \\
* & * & * & * & \varphi_{55} & 0 & \varphi_{i 57} & \varphi_{i 58} & \varphi_{i 59} \\
* & * & * & * & * & \varphi_{66} & \varphi_{i 67} & \varphi_{i 68} & \varphi_{i 69} \\
* & * & * & * & * & * & \varphi_{i 77} & \varphi_{i 78} & \varphi_{i 79} \\
* & * & * & * & * & * & * & \varphi_{i 88} & \varphi_{i 89} \\
* & * & * & * & * & * & * & * & \varphi_{i 99}
\end{array}\right]_{9 \times 9}
$$$$
\Psi_{i 12}=\left[\begin{array}{ccccccc}
0 & 0 & 0 & 0 & \varphi_{1,14} & 0 & \varphi_{i 1,16} \\
0 & 0 & 0 & 0 & 0 & 0 & \varphi_{i 2,16} \\
0 & 0 & 0 & 0 & \varphi_{3,14} & 0 & 0 \\
0 & 0 & 0 & 0 & 0 & 0 & 0 \\
0 & 0 & 0 & 0 & 0 & 0 & 0 \\
0 & 0 & 0 & 0 & 0 & 0 & 0 \\
0 & 0 & 0 & 0 & \varphi_{7,14} & 0 & \varphi_{i 7,16} \\
0 & 0 & 0 & 0 & 0 & 0 & \varphi_{i 8,16} \\
0 & 0 & 0 & 0 & 0 & 0 & \varphi_{i 9,16}
\end{array}\right]_{9 \times 7}
$$

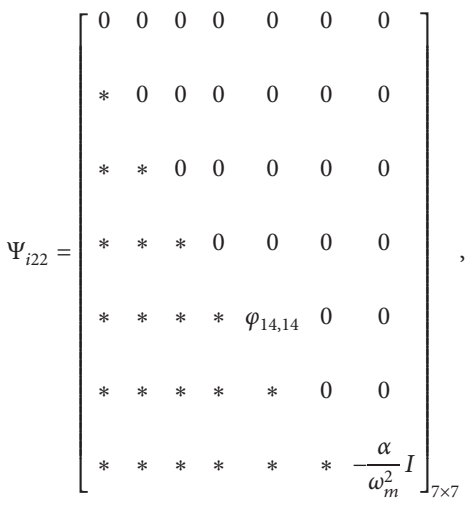

$\Psi_{i 13}=\left[\begin{array}{lllllllll}\varphi_{i 1,17} & \varphi_{i 2,17} & \varphi_{i 3,17} & \varphi_{i 4,17} & \varphi_{i 5,17} & \varphi_{i 6,17} & \varphi_{i 7,17} & \varphi_{i 8,17} & \varphi_{i 9,17}\end{array}\right]^{T}$,

$\Psi_{i 14}=\left[\begin{array}{lllllllll}\varepsilon E_{i a} & 0 & 0 & \varepsilon E_{i b} & 0 & 0 & 0 & 0 & 0\end{array}\right]^{T}$,

$\Psi_{i 23}=\left[\begin{array}{lllllll}0 & 0 & 0 & 0 & 0 & 0 & \varphi_{i 16,17}\end{array}\right]^{T}$,

$\Psi_{i 24}=\left[\begin{array}{lllllll}0 & 0 & 0 & 0 & 0 & 0 & \varepsilon E_{i d}\end{array}\right]^{T}$, 


$$
\begin{aligned}
& \varphi_{i 11}=P_{i 11} A_{i}+A_{i}^{T} P_{i 11}^{T}+P_{i 15} A_{i}+A_{i}^{T} P_{i 15}^{T}+P_{i 12}+P_{i 12}^{T}+h_{1}^{2} R_{1}+\left(h_{m}-h_{1}\right)^{2} R_{2}+\left(h_{2}-h_{m}\right)^{2} \\
& R_{3}+Q_{1}+M_{1} A_{i}+A_{i}^{T} M_{1}^{T}+\alpha P_{i 11}-9 R_{4} e^{-\alpha h_{1}}+\sum_{j \in U_{k}^{i}} \lambda_{i j}\left(P_{j 11}-W_{i 11}\right) \text {, } \\
& \varphi_{i 12}=-M_{1}-P_{i 15}+A_{i}^{T} M_{2}^{T} \text {, } \\
& \varphi_{i 13}=P_{i 13}-P_{i 12}+3 R_{4} e^{-\alpha h_{1}} \text {, } \\
& \varphi_{i 14}=P_{i 11} B_{i}+P_{i 15} B_{i}+M_{1} B_{i} \text {, } \\
& \varphi_{i 15}=P_{i 14}-P_{i 13} \text {, } \\
& \varphi_{i 16}=-P_{i 14} \text {, } \\
& \varphi_{1,14}=60 R_{4} e^{-\alpha h_{1}} \text {, } \\
& \varphi_{i 24}=M_{2} B_{i}, \\
& \varphi_{i 2,16}=M_{2} D_{i} \text {, } \\
& \varphi_{i 68}=-P_{i 34}^{T} \text {, } \\
& \varphi_{i 17}=A_{i}^{T} P_{i 21}^{T}+A_{i}^{T} P_{i 25}^{T}+P_{i 22}^{T}-\frac{24}{h_{1}} R_{4} e^{-\alpha h_{1}}+\alpha P_{i 12}+\sum_{j \in U_{k}^{i}} \lambda_{i j}\left(P_{j 12}-W_{i 12}\right) \text {, } \\
& \varphi_{i 18}=A_{i}^{T} P_{i 31}^{T}+A_{i}^{T} P_{i 35}^{T}+P_{i 23}+\alpha P_{i 13}+\sum_{j \in U_{k}^{i}} \lambda_{i j}\left(P_{j 13}-W_{i 13}\right), \\
& \varphi_{33}=e^{-\alpha h_{1}}\left(Q_{2}-Q_{1}\right)-9 R_{4} e^{-\alpha h_{1}}, \\
& \varphi_{i 19}=A_{i}^{T} P_{i 41}^{T}+A_{i}^{T} P_{i 45}^{T}+P_{i 24}+\alpha P_{i 14}+\sum_{j \in U_{k}^{i}} \lambda_{i j}\left(P_{j 14}-W_{i 14}\right) \text {, } \\
& \varphi_{i 37}=P_{i 23}^{T}-P_{i 22}^{T}+\frac{36}{h_{1}} R_{4} e^{-\alpha h_{1}}, \\
& \varphi_{i 1,16}=P_{i 11} D_{i}+P_{i 15} D_{i}+M_{1} D_{i}, \\
& \varphi_{i 88}=-e^{-\alpha h_{m}} R_{2}+\alpha P_{i 33}+\sum_{j \in U_{k}^{i}} \lambda_{i j}\left(P_{j 33}-W_{i 33}\right) \text {, } \\
& \varphi_{i 1,17}=P_{i 51}^{T}+P_{i 11} L_{i}+P_{i 51} L_{i}+M_{1} L_{i}+A_{i}^{T} P_{i 51}^{T}+A_{i} P_{i 55}-A_{i}^{T} P_{i 15}-P_{i 25} \text {, } \\
& \varphi_{22}=-M_{2}-M_{2}^{T}+h_{1}^{2} R_{4}+\left(h_{m}-h_{1}\right)^{2} R_{5}+\left(h_{2}-h_{m}\right)^{2} R_{6} \text {, } \\
& \varphi_{i 38}=P_{i 33}^{T}-P_{i 32}^{T} \text {, } \\
& \varphi_{i 39}=P_{i 43}^{T}-P_{i 42}^{T} \text {, } \\
& \varphi_{i 27}=-P_{i 25}^{T} \text {, } \\
& \varphi_{i 28}=-P_{i 35}^{T} \text {, } \\
& \varphi_{i 29}=-P_{i 45}^{T} \text {, } \\
& \varphi_{i 2,17}=M_{2} L_{i}-P_{i 55}^{T} \text {, } \\
& \varphi_{3,14}=-60 R_{4} e^{-\alpha h_{1}} \text {, }
\end{aligned}
$$




$$
\begin{aligned}
& \varphi_{i 47}=B_{i}^{T} P_{i 21}^{T}+B_{i}^{T} P_{i 25}^{T}, \\
& \varphi_{i 48}=B_{i}^{T} P_{i 31}^{T}+B_{i}^{T} P_{i 35}^{T}, \\
& \varphi_{i 49}=B_{i}^{T} P_{i 41}^{T}+B_{i}^{T} P_{i 45}^{T}, \\
& \varphi_{i 4,17}=B_{i}^{T} P_{i 51}^{T}+B_{i}^{T} P_{i 55}^{T}-B_{i}^{T} P_{i 15} \text {, } \\
& \varphi_{55}=e^{-\alpha h_{m}}\left(Q_{3}-Q_{2}\right), \\
& \varphi_{i 57}=P_{i 24}^{T}-P_{i 23}^{T} \text {, } \\
& \varphi_{i 58}=P_{i 34}^{T}-P_{i 33}^{T} \text {, } \\
& \varphi_{i 59}=P_{i 44}^{T}-P_{i 43}^{T} \text {, } \\
& \varphi_{i 66}=-e^{-\alpha h_{2}} Q_{3} \text {, } \\
& \varphi_{i 67}=-P_{i 24}^{T} \text {, } \\
& \varphi_{i 69}=-P_{i 44}^{T} \text {, } \\
& \varphi_{i 77}=-e^{-\alpha h_{1}} R_{1}+\alpha P_{i 22}-\frac{192}{h_{1}^{2}} R_{4} e^{-\alpha h_{1}}+\sum_{j \in U_{k}^{i}} \lambda_{i j}\left(P_{j 22}-W_{i 22}\right), \\
& \varphi_{i 78}=\alpha P_{i 23}+\sum_{j \in U_{k}^{i}} \lambda_{i j}\left(P_{j 23}-W_{i 23}\right) \text {, } \\
& \varphi_{14,14}=-720 R_{4} e^{-\alpha h_{1}}, \\
& \varphi_{i 79}=\alpha P_{i 24}+\sum_{j \in U_{k}^{i}} \lambda_{i j}\left(P_{j 24}-W_{i 24}\right), \\
& \varphi_{7,14}=\frac{360}{h_{1}} R_{4} e^{-\alpha h_{1}}, \\
& \varphi_{i 89}=\alpha P_{i 34}+\sum_{j \in U_{k}^{i}} \lambda_{i j}\left(P_{j 34}-W_{i 34}\right), \\
& \varphi_{i 99}=-e^{-\alpha h_{2}} R_{3}+\alpha P_{i 44}+\sum_{j \in U_{k}^{i}} \lambda_{i j}\left(P_{j 44}-W_{i 44}\right), \\
& \varphi_{i 7,16}=P_{i 21} D_{i}+P_{i 25} D_{i}, \\
& \varphi_{i 8,16}=P_{i 31} D_{i}+P_{i 35} D_{i} \text {, } \\
& \varphi_{i 9,16}=P_{i 41} D_{i}+P_{i 45} D_{i} \text {, } \\
& \varphi_{i 7,17}=P_{i 21} L_{i}+P_{i 25} L_{i} \text {, } \\
& \varphi_{i 8,17}=P_{i 31} L_{i}+P_{i 35} L_{i} \text {, } \\
& \varphi_{i 16,17}=D_{i}^{T} P_{i 51}^{T}+D_{i}^{T} P_{i 55}^{T}-D_{i}^{T} P_{i 15} \text {, } \\
& \varphi_{i 9,17}=P_{i 41} L_{i}+P_{i 45} L_{i}, \\
& \varphi_{i 17,17}=P_{i 51} L_{i}+L_{i}^{T} P_{i 51}^{T}+P_{i 55} L_{i}+L_{i}^{T} P_{i 55}^{T}-L_{i}-L_{i}^{T}-\varepsilon I, \\
& \widehat{\Psi}_{i}=\left[\varphi_{i, j}\right]_{16 \times 16^{\prime}} \\
& \Phi_{1}=\Phi^{(1)}+\Phi^{(2)}+\Phi^{(3)}+\Phi^{(4)}, \\
& \Phi_{2}=\widehat{\Phi}^{(1)}+\widehat{\Phi}^{(4)} \text {, } \\
& \Phi_{3}=\bar{\Phi}^{(2)}+\bar{\Phi}^{(4)} \text {, } \\
& \Pi_{1}=\Pi^{(1)}+\Pi^{(2)}+\Pi^{(3)}+\Pi^{(4)}, \\
& \Pi_{2}=\widehat{\Pi}^{(2)}+\widehat{\Pi}^{(4)} \text {, }
\end{aligned}
$$


where

$$
\begin{aligned}
& \Phi^{(1)}=\left[\phi_{i j}^{(1)}\right]_{18 \times 18}, \Phi^{(2)}=\left[\phi_{i j}^{(2)}\right]_{18 \times 18}, \Phi^{(3)}=\left[\phi_{i j}^{(3)}\right]_{18 \times 18}, \Phi^{(4)}=\left[\phi_{i j}^{(4)}\right]_{18 \times 18} \\
& \Pi^{(1)}=\left[\pi_{i j}^{(1)}\right]_{18 \times 18}, \Pi^{(2)}=\left[\pi_{i j}^{(2)}\right]_{18 \times 18}, \Pi^{(3)}=\left[\pi_{i j}^{(3)}\right]_{18 \times 18}, \widehat{\Phi}^{(1)}=\left[\phi_{i j}^{(1)}\right]_{16 \times 16} \\
& \widehat{\Phi}^{(2)}=\left[\phi_{i j}^{(2)}\right]_{16 \times 16}, \widehat{\Phi}^{(4)}=\left[\phi_{i j}^{(4)}\right]_{16 \times 16}, \bar{\Phi}^{(1)}=\left[\phi_{i j}^{(1)}\right]_{16 \times 16}, \bar{\Phi}^{(2)}=\left[\phi_{i j}^{(2)}\right]_{16 \times 16} \\
& \bar{\Phi}^{(4)}=\left[\phi_{i j}^{(4)}\right]_{16 \times 16}, \widehat{\Pi}^{(1)}=\left[\pi_{i j}^{(1)}\right]_{16 \times 16}, \widehat{\Pi}^{(2)}=\left[\pi_{i j}^{(2)}\right]_{16 \times 16}, \widehat{\Pi}^{(4)}=\left[\pi_{i j}^{(4)}\right]_{16 \times 16} .
\end{aligned}
$$

with

$$
\begin{aligned}
& \phi_{44}^{(1)}=-9 R_{5} e^{-\alpha h_{m}}, \phi_{45}^{(1)}=3 R_{5} e^{-\alpha h_{m}}, \phi_{4,10}^{(1)}=-24 R_{5} e^{-\alpha h_{m}}, \phi_{4,12}^{(1)}=60 R_{5} e^{-\alpha h_{m}}, \\
& \phi_{55}^{(1)}=-9 R_{5} e^{-\alpha h_{m}}, \phi_{5,10}^{(1)}=36 R_{5} e^{-\alpha h_{m}}, \phi_{5,12}^{(1)}=-60 R_{5} e^{-\alpha h_{m}}, \phi_{10,10}^{(1)}=-192 R_{5} e^{-\alpha h_{m}}, \\
& \phi_{10,12}^{(1)}=360 R_{5} e^{-\alpha h_{m}}, \phi_{12,12}^{(1)}=-720 R_{5} e^{-\alpha h_{m}}, \phi_{33}^{(2)}=-9 R_{5} e^{-\alpha h_{m}}, \phi_{34}^{(2)}=3 R_{5} e^{-\alpha h_{m}}, \\
& \phi_{3,11}^{(2)}=-24 R_{5} e^{-\alpha h_{m}}, \phi_{3,13}^{(2)}=60 R_{5} e^{-\alpha h_{m}}, \phi_{44}^{(2)}=-9 R_{5} e^{-\alpha h_{m}}, \phi_{4,11}^{(2)}=36 R_{5} e^{-\alpha h_{m}}, \\
& \phi_{4,13}^{(2)}=-60 R_{5} e^{-\alpha h_{m}}, \phi_{11,11}^{(2)}=-192 R_{5} e^{-\alpha h_{m}}, \phi_{11,13}^{(2)}=360 R_{5} e^{-\alpha h_{m}}, \phi_{13,13}^{(2)}=-720 R_{5} e^{-\alpha h_{m}}, \\
& \phi_{34}^{(3)}=-e^{-\alpha h_{m}}\left(X_{11}^{T}+X_{12}^{T}+X_{13}^{T}+X_{21}^{T}+X_{22}^{T}+X_{23}^{T}+X_{31}^{T}+X_{32}^{T}+X_{33}^{T}\right), \\
& \phi_{35}^{(3)}=e^{-\alpha h_{m}}\left(X_{11}^{T}+X_{12}^{T}+X_{13}^{T}-X_{21}^{T}-X_{22}^{T}-X_{23}^{T}+X_{31}^{T}+X_{32}^{T}+X_{33}^{T}\right), \\
& \phi_{3,10}^{(3)}=2 e^{-\alpha h_{m}}\left(X_{21}^{T}-3 X_{31}^{T}+X_{22}^{T}-3 X_{32}^{T}+X_{23}^{T}-3 X_{33}^{T}\right), \phi_{3,12}^{(3)}=12 e^{-\alpha h_{m}}\left(X_{31}^{T}+X_{32}^{T}+X_{33}^{T}\right), \\
& \phi_{44}^{(3)}=e^{-\alpha h_{m}}\left(\operatorname{sym}\left(X_{11}\right)+\operatorname{sym}\left(X_{21}\right)+\operatorname{sym}\left(X_{31}\right)-\operatorname{sym}\left(X_{12}\right)-\operatorname{sym}\left(X_{22}\right)\right. \\
& \left.-\operatorname{sym}\left(X_{32}\right)+\operatorname{sym}\left(X_{13}\right)+\operatorname{sym}\left(X_{23}\right)+\operatorname{sym}\left(X_{33}\right)\right) \text {, } \\
& \phi_{45}^{(3)}=-e^{-\alpha h_{m}}\left(X_{11}^{T}-X_{21}^{T}+X_{31}^{T}-X_{12}^{T}+X_{22}^{T}-X_{32}^{T}+X_{13}^{T}-X_{23}^{T}+X_{33} T\right) \text {, } \\
& \phi_{4,10}^{(3)}=-2 e^{-\alpha h_{m}}\left(X_{21}^{T}-3 X_{31}^{T}-X_{22}^{T}+3 X_{32}^{T}+X_{23}^{T}-3 X_{33}^{T}\right), \phi_{10,13}^{(3)}=-24 e^{-\alpha h_{m}}\left(X_{23}-3 X_{33}\right) \text {, } \\
& \phi_{4,11}^{(3)}=2 e^{-\alpha h_{m}}\left(X_{12}+X_{22}+X_{32}-3 X_{13}-3 X_{23}-3 X_{33}\right), \phi_{11,12}^{(3)}=-24 e^{-\alpha h_{m}}\left(X_{32}^{T}-3 X_{33}\right), \\
& \phi_{4,12}^{(3)}=-12 e^{-\alpha h_{m}}\left(X_{31}^{T}-X_{32}^{T}+X_{33}^{T}\right), \phi_{4,13}^{(3)}=12 e^{-\alpha h_{m}}\left(X_{13}+X_{23}+X_{33}\right), \\
& \phi_{5,13}^{(3)}=-12 e^{-\alpha h_{m}}\left(X_{13}-X_{23}+X_{33}\right), \phi_{10,11}^{(3)}=-4 e^{-\alpha h_{m}}\left(X_{22}-3 X_{32}-3 X_{23}+9 X_{33}\right) \text {, } \\
& \phi_{5,11}^{(3)}=-2 e^{-\alpha h_{m}}\left(X_{12}-X_{22}+X_{32}-3 X_{13}+3 X_{23}-3 X_{33}\right), \phi_{12,13}^{(3)}=-144 e^{-\alpha h_{m}}\left(X_{33}\right),
\end{aligned}
$$




$$
\begin{aligned}
& \phi_{55}^{(4)}=-9 R_{6} e^{-\alpha h_{2}}, \phi_{56}^{(4)}=3 R_{6} e^{-\alpha h_{2}}, \phi_{59}^{(4)}=-\frac{24}{h_{2}-h_{m}} R_{6} e^{-\alpha h_{2}}, \phi_{5,15}^{(4)}=60 R_{6} e^{-\alpha h_{2}}, \\
& \phi_{66}^{(4)}=-9 R_{6} e^{-\alpha h_{2}}, \phi_{69}^{(4)}=\frac{36}{h_{2}-h_{m}} R_{6} e^{-\alpha h_{2}}, \phi_{6,15}^{(4)}=-60 R_{6} e^{-\alpha h_{2}}, \phi_{99}^{(4)}=-\frac{192}{\left(h_{2}-h_{m}\right)^{2}} R_{6} e^{-\alpha h_{2}}, \\
& \phi_{9,15}^{(4)}=\frac{360}{h_{2}-h_{m}} R_{6} e^{-\alpha h_{2}}, \phi_{15,15}^{(4)}=-720 R_{6} e^{-\alpha h_{2}}, \pi_{44}^{(1)}=-9 R_{6} e^{-\alpha h_{2}}, \pi_{46}^{(1)}=3 R_{6} e^{-\alpha h_{2}}, \\
& \pi_{4,10}^{(1)}=-24 R_{6} e^{-\alpha h_{2}}, \pi_{4,12}^{(1)}=60 R_{6} e^{-\alpha h_{2}}, \pi_{66}^{(1)}=-9 R_{6} e^{-\alpha h_{2}}, \pi_{6,10}^{(1)}=36 R_{6} e^{-\alpha h_{2}}, \\
& \pi_{6,12}^{(1)}=-60 R_{6} e^{-\alpha h_{2}}, \pi_{10,10}^{(1)}=-192 R_{6} e^{-\alpha h_{2}}, \pi_{10,12}^{(1)}=360 R_{6} e^{-\alpha h_{2}}, \pi_{12,12}^{(1)}=-720 R_{6} e^{-\alpha h_{2}}, \\
& \pi_{44}^{(2)}=-9 R_{6} e^{-\alpha h_{2}}, \pi_{45}^{(2)}=3 R_{6} e^{-\alpha h_{2}}, \pi_{4,11}^{(2)}=-24 R_{6} e^{-\alpha h_{2}}, \pi_{4,13}^{(2)}=60 R_{6} e^{-\alpha h_{2}}, \\
& \pi_{55}^{(1)}=-9 R_{6} e^{-\alpha h_{2}}, \pi_{5,11}^{(2)}=36 R_{6} e^{-\alpha h_{2}}, \pi_{5,13}^{(2)}=-60 R_{6} e^{-\alpha h_{2}}, \\
& \pi_{11,11}^{(2)}=-192 R_{6} e^{-\alpha h_{2}}, \pi_{11,13}^{(2)}=360 R_{6} e^{-\alpha h_{2}}, \pi_{13,13}^{(2)}=-720 R_{6} e^{-\alpha h_{2}}, \\
& \pi_{44}^{(3)}=e^{-\alpha h_{2}}\left(\operatorname{sym}\left(Y_{11}\right)+\operatorname{sym}\left(Y_{21}\right)+\operatorname{sym}\left(Y_{31}\right)-\operatorname{sym}\left(Y_{12}\right)-\operatorname{sym}\left(Y_{22}\right)\right. \\
& \left.-\operatorname{sym}\left(Y_{32}\right)+\operatorname{sym}\left(Y_{13}\right)+\operatorname{sym}\left(Y_{23}\right)+\operatorname{sym}\left(Y_{33}\right)\right) \text {, } \\
& \pi_{45}^{(3)}=-e^{-\alpha h_{2}}\left(Y_{11}+Y_{21}+Y_{31}+Y_{12}+Y_{22}+Y_{32}+Y_{13}+Y_{23}+Y_{33}\right) \text {, } \\
& \pi_{46}^{(3)}=-e^{-\alpha h_{2}}\left(Y_{11}^{T}-Y_{21}^{T}+Y_{31}^{T}-Y_{12}^{T}+Y_{22}^{T}-Y_{32}^{T}+Y_{13}^{T}-Y_{23}^{T}+Y_{33}^{T}\right) \text {, } \\
& \pi_{4,10}^{(3)}=-e^{-\alpha h_{2}}\left(2 Y_{21}^{T}-6 Y_{31}^{T}-2 Y_{22}^{T}+6 Y_{32}^{T}+2 Y_{23}^{T}-6 Y_{33}^{T}\right), \pi_{4,12}^{(3)}=-12 e^{-\alpha h_{2}}\left(Y_{31}^{T}-Y_{32}^{T}+Y_{33}^{T}\right) \text {, } \\
& \pi_{4,11}^{(3)}=e^{-\alpha h_{2}}\left(2 Y_{12}+2 Y_{22}+2 Y_{32}-6 Y_{13}-6 Y_{23}-6 Y_{33}\right), \pi_{4,13}^{(3)}=12 e^{-\alpha h_{2}}\left(Y_{13}+Y_{23}+Y_{33}\right) \text {, } \\
& \pi_{56}^{(3)}=e^{-\alpha h_{2}}\left(Y_{11}^{T}-Y_{21}^{T}+Y_{31}^{T}+Y_{12}^{T}-Y_{22}^{T}+Y_{32}^{T}+Y_{13}^{T}-Y_{23}^{T}+Y_{33}^{T}\right) \text {, } \\
& \pi_{5,10}^{(3)}=2 e^{-\alpha h_{2}}\left(Y_{21}^{T}-3 Y_{31}^{T}+Y_{22}^{T}-3 Y_{32}^{T}+Y_{23}^{T}-3 Y_{33}^{T}\right), \pi_{5,12}^{(3)}=12 e^{-\alpha h_{2}}\left(Y_{31}^{T}+Y_{32}^{T}+Y_{33}^{T}\right) \text {, } \\
& \pi_{6,11}^{(3)}=-2 e^{-\alpha h_{2}}\left(Y_{12}-Y_{22}+Y_{32}-3 Y_{13}+3 Y_{23}-3 Y_{33}\right), \pi_{6,13}^{(3)}=-12 e^{-\alpha h_{2}}\left(Y_{13}-Y_{23}+Y_{33}\right) \text {, } \\
& \pi_{10,11}^{(3)}=-e^{-\alpha h_{2}}\left(4 Y_{22}-12 Y_{32}-12 Y_{23}+36 Y_{33}\right), \pi_{10,13}^{(3)}=24 e^{-\alpha h_{2}}\left(3 Y_{33}-Y_{23}\right) \text {, } \\
& \pi_{11,12}^{(3)}=24 e^{-\alpha h_{2}}\left(3 Y_{33}^{T}-Y_{32}^{T}\right), \pi_{12,13}^{(3)}=-144 e^{-\alpha h_{2}}\left(Y_{33}\right) \text {, } \\
& \pi_{33}^{(4)}=-9 R_{5} e^{-\alpha h_{m}}, \pi_{35}^{(4)}=3 R_{5} e^{-\alpha h_{m}}, \pi_{38}^{(4)}=-\frac{24}{h_{m}-h_{1}} R_{5} e^{-\alpha h_{m}}, \pi_{3,15}^{(4)}=60 R_{5} e^{-\alpha h_{m}}, \\
& \pi_{55}^{(4)}=-9 R_{5} e^{-\alpha h_{m}}, \pi_{58}^{(4)}=\frac{36}{h_{m}-h_{1}} R_{5} e^{-\alpha h_{m}}, \pi_{5,15}^{(4)}=-60 R_{5} e^{-\alpha h_{m}}, \\
& \pi_{88}^{(4)}=-\frac{192}{\left(h_{m}-h_{1}\right)^{2}} R_{5} e^{-\alpha h_{m}}, \pi_{8,15}^{(4)}=\frac{360}{h_{m}-h_{1}} R_{5} e^{-\alpha h_{m}}, \pi_{15,15}^{(4)}=-720 R_{5} e^{-\alpha h_{m}}, \\
& \widehat{\phi}_{44}^{(1)}=-9 R_{5} e^{-\alpha h_{m}}, \widehat{\phi}_{45}^{(1)}=3 R_{5} e^{-\alpha h_{m}}, \widehat{\phi}_{4,10}^{(1)}=-24 R_{5} e^{-\alpha h_{m}}, \widehat{\phi}_{4,11}^{(1)}=60 R_{5} e^{-\alpha h_{m}} \text {, } \\
& \widehat{\phi}_{55}^{(1)}=-9 R_{5} e^{-\alpha h_{m}}, \widehat{\phi}_{5,10}^{(1)}=36 R_{5} e^{-\alpha h_{m}}, \widehat{\phi}_{5,11}^{(1)}=-60 R_{5} e^{-\alpha h_{m}}, \widehat{\phi}_{10,10}^{(1)}=-192 R_{5} e^{-\alpha h_{m}} \text {, } \\
& \widehat{\phi}_{10,11}^{(1)}=360 R_{5} e^{-\alpha h_{m}}, \widehat{\phi}_{11,11}^{(1)}=-720 R_{5} e^{-\alpha h_{m}}, \widehat{\phi}_{55}^{(4)}=-9 R_{6} e^{-\alpha h_{2}}, \widehat{\phi}_{56}^{(4)}=3 R_{6} e^{-\alpha h_{2}} \text {, }
\end{aligned}
$$




$$
\begin{aligned}
& \widehat{\phi}_{59}^{(4)}=-\frac{24}{h_{2}-h_{m}} R_{6} e^{-\alpha h_{2}}, \widehat{\phi}_{5,13}^{(4)}=60 R_{6} e^{-\alpha h_{2}}, \widehat{\phi}_{66}^{(4)}=-9 R_{6} e^{-\alpha h_{2}}, \widehat{\phi}_{69}^{(4)}=\frac{36}{h_{2}-h_{m}} R_{6} e^{-\alpha h_{2}}, \\
& \widehat{\phi}_{6,13}^{(4)}=-60 R_{6} e^{-\alpha h_{2}}, \widehat{\phi}_{99}^{(4)}=-\frac{192}{\left(h_{2}-h_{m}\right)^{2}} R_{6} e^{-\alpha h_{2}}, \widehat{\phi}_{9,13}^{(4)}=\frac{360}{h_{2}-h_{m}} R_{6} e^{-\alpha h_{2}}, \widehat{\phi}_{13,13}^{(4)}=-720 R_{6} e^{-\alpha h_{2}} \text {, } \\
& \bar{\phi}_{33}^{(2)}=-9 R_{5} e^{-\alpha h_{m}}, \bar{\phi}_{34}^{(2)}=3 R_{5} e^{-\alpha h_{m}}, \bar{\phi}_{3,10}^{(2)}=-24 R_{5} e^{-\alpha h_{m}}, \bar{\phi}_{3,11}^{(2)}=60 R_{5} e^{-\alpha h_{m}}, \bar{\phi}_{44}^{(2)}=-9 R_{5} e^{-\alpha h_{m}} \text {, } \\
& \bar{\phi}_{4,10}^{(2)}=36 R_{5} e^{-\alpha h_{m}}, \bar{\phi}_{4,11}^{(2)}=-60 R_{5} e^{-\alpha h_{m}}, \bar{\phi}_{10,10}^{(2)}=-192 R_{5} e^{-\alpha h_{m}}, \bar{\phi}_{10,11}^{(2)}=360 R_{5} e^{-\alpha h_{m}} \text {, } \\
& \bar{\phi}_{11,11}^{(2)}=-720 R_{5} e^{-\alpha h_{m}}, \bar{\phi}_{55}^{(4)}=-9 R_{6} e^{-\alpha h_{2}}, \bar{\phi}_{56}^{(4)}=3 R_{6} e^{-\alpha h_{2}}, \bar{\phi}_{59}^{(4)}=-\frac{24}{h_{2}-h_{m}} R_{6} e^{-\alpha h_{2}} \text {, } \\
& \widehat{\phi}_{5,13}^{(4)}=60 R_{6} e^{-\alpha h_{2}}, \bar{\phi}_{66}^{(4)}=-9 R_{6} e^{-\alpha h_{2}}, \bar{\phi}_{69}^{(4)}=\frac{36}{h_{2}-h_{m}} R_{6} e^{-\alpha h_{2}}, \bar{\phi}_{6,13}^{(4)}=-60 R_{6} e^{-\alpha h_{2}} \text {, } \\
& \bar{\phi}_{99}^{(4)}=-\frac{192}{\left(h_{2}-h_{m}\right)^{2}} R_{6} e^{-\alpha h_{2}}, \bar{\phi}_{9,13}^{(4)}=\frac{360}{h_{2}-h_{m}} R_{6} e^{-\alpha h_{2}}, \bar{\phi}_{13,13}^{(4)}=-720 R_{6} e^{-\alpha h_{2}}, \\
& \widehat{\pi}_{44}^{(2)}=-9 R_{6} e^{-\alpha h_{2}}, \widehat{\pi}_{45}^{(2)}=3 R_{6} e^{-\alpha h_{2}}, \widehat{\pi}_{4,10}^{(2)}=-24 R_{6} e^{-\alpha h_{2}}, \widehat{\pi}_{4,11}^{(2)}=60 R_{6} e^{-\alpha h_{2}}, \widehat{\pi}_{55}^{(2)}=-9 R_{6} e^{-\alpha h_{2}}, \\
& \widehat{\pi}_{5,10}^{(2)}=36 R_{6} e^{-\alpha h_{2}}, \widehat{\pi}_{5,11}^{(2)}=-60 R_{6} e^{-\alpha h_{2}}, \widehat{\pi}_{10,10}^{(2)}=-192 R_{6} e^{-\alpha h_{2}}, \widehat{\pi}_{10,11}^{(2)}=360 R_{6} e^{-\alpha h_{2}} \text {, } \\
& \widehat{\pi}_{11,11}^{(2)}=-720 R_{6} e^{-\alpha h_{2}}, \widehat{\pi}_{33}^{(4)}=-9 R_{5} e^{-\alpha h_{m}}, \widehat{\pi}_{35}^{(4)}=3 R_{5} e^{-\alpha h_{m}}, \widehat{\pi}_{38}^{(4)}=-\frac{24}{h_{m}-h_{1}} R_{5} e^{-\alpha h_{m}} \text {, } \\
& \widehat{\pi}_{3,13}^{(4)}=60 R_{5} e^{-\alpha h_{m}}, \widehat{\pi}_{55}^{(4)}=-9 R_{5} e^{-\alpha h_{m}}, \widehat{\pi}_{58}^{(4)}=\frac{36}{h_{m}-h_{1}} R_{5} e^{-\alpha h_{m}}, \widehat{\pi}_{5,13}^{(4)}=-60 R_{5} e^{-\alpha h_{m}}, \\
& \widehat{\pi}_{88}^{(4)}=-\frac{192}{\left(h_{m}-h_{1}\right)^{2}} R_{5} e^{-\alpha h_{m}}, \widehat{\pi}_{8,13}^{(4)}=\frac{360}{h_{m}-h_{1}} R_{5} e^{-\alpha h_{m}}, \widehat{\pi}_{13,13}^{(4)}=-720 R_{5} e^{-\alpha h_{m}} .
\end{aligned}
$$

The other elements in $\Phi^{(1)}, \Phi^{(2)}, \Phi^{(3)}, \Phi^{(4)}, \Pi^{(1)}, \Pi^{(2)}$, $\Pi^{(3)}, \Pi^{(4)}, \widehat{\Phi}^{(2)}, \widehat{\Phi}^{(4)}, \Phi^{(1)}, \Phi^{(2)}, \Phi^{(4)}, \widehat{\Pi}^{(1)}, \widehat{\Pi}^{(2)}$, and $\widehat{\Pi}^{\prime(4)}$ are equal to zero.

\section{B. The Representation of $\Psi_{i}, \widehat{\Psi}_{i}, \Phi_{1}, \Phi_{2}$} $\Phi_{3}, \Pi_{1}, \Pi_{2}$ in Corollary 1

$$
\Psi_{i}=\left[\begin{array}{cccc}
\Psi_{i 11} & \Psi_{i 12} & \Psi_{i 13} & \Psi_{i 14} \\
* & \Psi_{i 22} & \Psi_{i 23} & \Psi_{i 24} \\
* & * & \varphi_{i 17,17} & 0 \\
* & * & * & -\varepsilon I
\end{array}\right]
$$

$$
\begin{aligned}
\varphi_{i 11}= & P_{i 11} A_{i}+A_{i}^{T} P_{i 11}^{T}+P_{i 15} A_{i}+A_{i}^{T} P_{i 15}^{T}+P_{i 12}+P_{i 12}^{T}+h_{1}^{2} R_{1}+\left(h_{m}-h_{1}\right)^{2} R_{2}+\left(h_{2}-h_{m}\right)^{2} \\
& R_{3}+Q_{1}+M_{1} A_{i}+A_{i}^{T} M_{1}^{T}+\alpha P_{i 11}-9 R_{4} e^{-\alpha h_{1}}+\sum_{j \in U_{k}^{i}} \lambda_{i j} P_{j 11}, \\
\varphi_{i 17}= & A_{i}^{T} P_{i 21}^{T}+A_{i}^{T} P_{i 25}^{T}+P_{i 22}^{T}-\frac{24}{h_{1}} R_{4} e^{-\alpha h_{1}}+\alpha P_{i 12}+\sum_{j \in U_{k}^{i}} \lambda_{i j} P_{j 12}, \\
\varphi_{i 18}= & A_{i}^{T} P_{i 31}^{T}+A_{i}^{T} P_{i 35}^{T}+P_{i 23}+\alpha P_{i 13}+\sum_{j \in U_{k}^{i}} \lambda_{i j} P_{j 13},
\end{aligned}
$$




$$
\begin{aligned}
& \varphi_{i 19}=A_{i}^{T} P_{i 41}^{T}+A_{i}^{T} P_{i 45}^{T}+P_{i 24}+\alpha P_{i 14}+\sum_{j \in U_{k}^{i}} \lambda_{i j} P_{j 14} \\
& \varphi_{i 77}=-e^{-\alpha h_{1}} R_{1}+\alpha P_{i 22}-\frac{192}{h_{1}^{2}} R_{4} e^{-\alpha h_{1}}+\sum_{j \in U_{k}^{i}} \lambda_{i j} P_{j 22} \\
& \varphi_{i 78}=\alpha P_{i 23}+\sum_{j \in U_{k}^{i}} \lambda_{i j} P_{j 23} \\
& \varphi_{i 79}=\alpha P_{i 24}+\sum_{j \in U_{k}^{i}} \lambda_{i j} P_{j 24}, \\
& \varphi_{i 88}=-e^{-\alpha h_{m}} R_{2}+\alpha P_{i 33}+\sum_{j \in U_{k}^{i}} \lambda_{i j} P_{j 33} \\
& \varphi_{i 89}=\alpha P_{i 34}+\sum_{j \in U_{k}^{i}} \lambda_{i j} P_{j 34}, \\
& \varphi_{i 99}=-e^{-\alpha h_{2}} R_{3}+\alpha P_{i 44}+\sum_{j \in U_{k}^{i}} \lambda_{i j} P_{j 44} .
\end{aligned}
$$

The other elements are the same as those defined in Appendix A.

C. The Representation of $\Psi_{i}, \widehat{\Psi}_{i}, \Phi_{1}, \Phi_{2}$, $\Phi_{3}, \Pi_{1}, \Pi_{2}$ in Corollary 2

$$
\Psi_{i}=\left[\begin{array}{cccc}
\Psi_{i 11} & \Psi_{i 12} & \Psi_{i 13} & \Psi_{i 14} \\
* & \Psi_{i 22} & \Psi_{i 23} & \Psi_{i 24} \\
* & * & \varphi_{i 17,17} & 0 \\
* & * & * & -\varepsilon I
\end{array}\right]
$$

where

$$
\begin{aligned}
\varphi_{i 11}= & P_{i 11} A_{i}+A_{i}^{T} P_{i 11}^{T}+P_{i 15} A_{i}+A_{i}^{T} P_{i 15}^{T}+P_{i 12}+P_{i 12}^{T}+h_{1}^{2} R_{1}+\left(h_{m}-h_{1}\right)^{2} R_{2}+\left(h_{2}-h_{m}\right)^{2} \\
& R_{3}+Q_{1}+M_{1} A_{i}+A_{i}^{T} M_{1}^{T}+\alpha P_{i 11}-9 R_{4} e^{-\alpha h_{1}}, \\
\varphi_{i 17}= & A_{i}^{T} P_{i 21}^{T}+A_{i}^{T} P_{i 25}^{T}+P_{i 22}^{T}-\frac{24}{h_{1}} R_{4} e^{-\alpha h_{1}}+\alpha P_{i 12}, \\
\varphi_{i 18}= & A_{i}^{T} P_{i 31}^{T}+A_{i}^{T} P_{i 35}^{T}+P_{i 23}+\alpha P_{i 13}, \\
\varphi_{i 19}= & A_{i}^{T} P_{i 41}^{T}+A_{i}^{T} P_{i 45}^{T}+P_{i 24}+\alpha P_{i 14}, \\
\varphi_{i 77}= & -e^{-\alpha h_{1}} R_{1}+\alpha P_{i 22}-\frac{192}{h_{1}^{2}} R_{4} e^{-\alpha h_{1}}, \\
\varphi_{i 78}= & \alpha P_{i 23}, \\
\varphi_{i 79}= & \alpha P_{i 24}, \\
\varphi_{i 88}= & -e^{-\alpha h_{m}} R_{2}+\alpha P_{i 33}, \\
\varphi_{i 89}= & \alpha P_{i 34}, \\
\varphi_{i 99}= & -e^{-\alpha h_{2}} R_{3}+\alpha P_{i 44} .
\end{aligned}
$$


The other elements are the same as those defined in Appendix A.

D. The Representation of $\Psi_{i}, \widehat{\Psi}_{i}, \Phi_{1}$, $\Phi_{2}, \Phi_{3}, \Pi_{1}, \Pi_{2}$ in Theorem 2

$$
\Psi_{i}=\left[\begin{array}{cc}
\widetilde{\Psi}_{i 11} & \Psi_{i 12} \\
* & \Psi_{i 22}
\end{array}\right],
$$

where

$$
\begin{aligned}
& \widetilde{\Psi}_{i 11}=\left[\begin{array}{ccccccccc}
\varphi_{i 11} & \varphi_{i 12} & \varphi_{i 13} & \varphi_{i 14} & \varphi_{i 15} & \varphi_{i 16} & \varphi_{i 17} & \varphi_{i 18} & \varphi_{i 19} \\
* & \varphi_{22} & 0 & \varphi_{i 24} & 0 & 0 & 0 & 0 & 0 \\
* & * & \varphi_{33} & 0 & 0 & 0 & \varphi_{i 37} & \varphi_{i 38} & \varphi_{i 39} \\
* & * & * & 0 & 0 & 0 & \varphi_{i 47} & \varphi_{i 48} & \varphi_{i 49} \\
* & * & * & * & \varphi_{55} & 0 & \varphi_{i 57} & \varphi_{i 58} & \varphi_{i 59} \\
* & * & * & * & * & \varphi_{66} & \varphi_{i 67} & \varphi_{i 68} & \varphi_{i 69} \\
* & * & * & * & * & * & \varphi_{i 77} & \varphi_{i 78} & \varphi_{i 79} \\
* & * & * & * & * & * & * & \varphi_{i 88} & \varphi_{i 89} \\
* & * & * & * & * & * & * & * & \varphi_{i 99}
\end{array}\right]_{9 \times 9} \\
& \varphi_{i 11}=P_{i 11} A_{i}+A_{i}^{T} P_{i 11}^{T}+P_{i 12}+P_{i 12}^{T}+h_{1}^{2} R_{1}+\left(h_{m}-h_{1}\right)^{2} R_{2}+\left(h_{2}-h_{m}\right)^{2} R_{3}+M_{1} A_{i}+A_{i}^{T} M_{1}^{T} \\
& +Q_{1}+\alpha P_{i 11}-9 R_{4} e^{-\alpha h_{1}}+\sum_{j \in U_{k}^{i}} \lambda_{i j}\left(P_{j 11}-W_{i 11}\right) \\
& \varphi_{i 12}=-M_{1}+A_{i}^{T} M_{2}^{T}, \\
& \varphi_{i 14}=P_{i 11} B_{i}+M_{1} B_{i} \text {, } \\
& \varphi_{i 18}=A_{i}^{T} P_{i 31}^{T}+P_{i 23}+\alpha P_{i 13}+\sum_{j \in U_{k}^{i}} \lambda_{i j}\left(P_{j 13}-W_{i 13}\right) \text {, } \\
& \varphi_{i 17}=A_{i}^{T} P_{i 21}^{T}+P_{i 22}^{T}-\frac{24}{h_{1}} R_{4} e^{-\alpha h_{1}}+\alpha P_{i 12}+\sum_{j \in U_{k}^{i}} \lambda_{i j}\left(P_{j 12}-W_{i 12}\right), \\
& \varphi_{i 1,16}=P_{i 11} D_{i}+M_{1} D_{i} \text {, } \\
& \varphi_{i 19}=A_{i}^{T} P_{i 41}^{T}+P_{i 24}+\alpha P_{i 14}+\sum_{j \in U_{k}^{i}} \lambda_{i j}\left(P_{j 14}-W_{i 14}\right) \\
& \varphi_{i 47}=B_{i}^{T} P_{i 21}^{T}, \\
& \varphi_{i 48}=B_{i}^{T} P_{i 31}^{T} \text {, } \\
& \varphi_{i 49}=B_{i}^{T} P_{i 41}^{T}, \\
& \varphi_{i 7,16}=P_{i 21} D_{i} \text {, } \\
& \varphi_{i 8,16}=P_{i 31} D_{i} \text {, } \\
& \varphi_{i 9,16}=P_{i 41} D_{i} \text {, }
\end{aligned}
$$

The other elements are defined in Appendix A. 
E. The Representation of $\Psi_{i}, \widehat{\Psi}_{i}, \Phi_{1}, \Phi_{2}$, $\Phi_{3}, \Pi_{1}, \Pi_{2}$ in Corollary 3

$$
\Psi_{i}=\left[\begin{array}{cc}
\widetilde{\Psi}_{i 11} & \Psi_{i 12} \\
* & \Psi_{i 22}
\end{array}\right],
$$

where

$$
\begin{aligned}
& \varphi_{i 11}=\alpha P_{i 11}+P_{i 11} A_{i}+A_{i}^{T} P_{i 11}^{T}+P_{i 12}+P_{i 12}^{T}+h_{1}^{2} R_{1} \\
& +\left(h_{m}-h_{1}\right)^{2} R_{2}+\left(h_{2}-h_{m}\right)^{2} \\
& R_{3}+Q_{1}-9 R_{4} e^{-\alpha h_{1}}+M_{1} A_{i}+A_{i}^{T} M_{1}^{T}+\sum_{j \in U_{k}^{i}} \lambda_{i j} P_{j 11}, \\
& \varphi_{i 17}=A_{i}^{T} P_{i 21}^{T}+P_{i 22}^{T}-\frac{24}{h_{1}} R_{4} e^{-\alpha h_{1}}+\alpha P_{i 12}+\sum_{j \in U_{k}^{i}} \lambda_{i j} P_{j 12} \\
& \varphi_{i 18}=A_{i}^{T} P_{i 31}^{T}+P_{i 23}+\alpha P_{i 13}+\sum_{j U_{k}^{i}} \lambda_{i j} P_{j 13}, \\
& \varphi_{i 19}=A_{i}^{T} P_{i 41}^{T}+P_{i 24}+\alpha P_{i 14}+\sum_{j U_{k}^{i}} \lambda_{i j} P_{j 14}, \\
& \varphi_{i 77}=-e^{-\alpha h_{1}} R_{1}+\alpha P_{i 22}-\frac{192}{h_{1}^{2}} R_{4} e^{-\alpha h_{1}}+\sum_{j U_{k}^{i}} \lambda_{i j} P_{j 22}, \\
& \varphi_{i 78}=\alpha P_{i 23}+\sum_{j \in U_{k}^{i}} \lambda_{i j} P_{j 23}, \\
& \varphi_{i 79}=\alpha P_{i 24}+\sum_{j \in U_{k}^{i}} \lambda_{i j} P_{j 24}, \\
& \varphi_{i 99}=-e^{-\alpha h_{2}} R_{3}+\alpha P_{i 44}+\sum_{j \in U_{k}^{i}} \lambda_{i j} P_{j 44}, \\
& \varphi_{i 88}=-e^{-\alpha h_{m}} R_{2}+\alpha P_{i 33}+\sum_{j \in U_{k}^{i}} \lambda_{i j} P_{j 33}, \\
& \varphi_{i 89}=\alpha P_{i 34}+\sum_{j \in U_{k}^{i}} \lambda_{i j} P_{j 34}
\end{aligned}
$$

The elements $\widetilde{\Psi}_{i 11} \quad \Psi_{i 12} \quad \Psi_{i 22}$ and other $\varphi$ are the same as those defined in Appendix D.

\section{F. The Representation of $\Psi_{i}, \widehat{\Psi}_{i}, \Phi_{1}, \Phi_{2}$, $\Phi_{3}, \Pi_{1}, \Pi_{2}$ in Corollary 4}

$$
\Psi_{i}=\left[\begin{array}{cc}
\widetilde{\Psi}_{i 11} & \Psi_{i 12} \\
* & \Psi_{i 22}
\end{array}\right],
$$

where

$$
\begin{aligned}
\varphi_{i 11}= & \alpha P_{i 11}+P_{i 11} A_{i}+A_{i}^{T} P_{i 11}^{T}+P_{i 12}+P_{i 12}^{T}+h_{1}^{2} R_{1} \\
& +\left(h_{m}-h_{1}\right)^{2} R_{2}+Q_{1}+\left(h_{2}-h_{m}\right)^{2} R_{3}-9 R_{4} e^{-\alpha} \\
& +M_{1} A_{i}+A_{i}^{T} M_{1}^{T}, \\
\varphi_{i 17}= & A_{i}^{T} P_{i 21}^{T}+P_{i 22}^{T}-\frac{24}{h_{1}} R_{4} e^{-\alpha h_{1}}+\alpha P_{i 12}, \\
\varphi_{i 89}= & \alpha P_{i 34}, \\
\varphi_{i 18}= & A_{i}^{T} P_{i 31}^{T}+P_{i 23}+\alpha P_{i 13}, \\
\varphi_{i 19}= & A_{i}^{T} P_{i 41}^{T}+P_{i 24}+\alpha P_{i 14}, \\
\varphi_{i 77}= & -e^{-\alpha h_{1}} R_{1}+\alpha P_{i 22}-\frac{192}{h_{1}^{2}} R_{4} e^{-\alpha h_{1}}, \\
\varphi_{i 78}= & \alpha P_{i 23}, \\
\varphi_{i 79}= & \alpha P_{i 24}, \\
\varphi_{i 88}= & -e^{-\alpha h_{m}} R_{2}+\alpha P_{i 33}, \\
\varphi_{i 99}= & -e^{-\alpha h_{2}} R_{3}+\alpha P_{i 44} .
\end{aligned}
$$$$
+\left(h_{m}-h_{1}\right)^{2} R_{2}+Q_{1}+\left(h_{2}-h_{m}\right)^{2} R_{3}-9 R_{4} e^{-\alpha h_{1}}
$$

The other elements are the same as those defined in Appendix D.

\section{Data Availability}

As a research paper, this paper mainly studies the theory of dynamic properties of time-delay differential system, and some numerical simulations were carried out by MATLAB. The data and program used to support the findings of this study are available from the corresponding author upon request.

\section{Conflicts of Interest}

The authors declare that they have no conflicts of interest.

\section{Acknowledgments}

This research was supported by the Science and Technology Foundation of Guizhou Province (nos. LKM[2013]21, J [2015]2074, and J[2016]1074), Doctoral Fund Project of Guizhou Minzu University (no. 006), and Project for Young Talents Growth of Guizhou Provincial Department of Education (no. Ky[2017]133).

\section{References}

[1] Z.-Y. Li, J. Lam, and Y. Wang, "Stability analysis of linear stochastic neutral-type time-delay systems with two delays," Automatica, vol. 91, pp. 179-189, 2018. 
[2] S. Long, S. Zhong, H. Guan, and D. Zhang, "Exponential stability analysis for a class of neutral singular markovian jump systems with time-varying delays," Journal of the Franklin Institute, vol. 356, no. 12, pp. 6015-6040, 2019.

[3] W. H. Qi, X. Yang, X. W. Gao, J. Cheng, Y. G. Kao, and Y. L. Wei, "Stability for delayed switched systems with markov jump parameters and generally incomplete transition rates," Applied Mathematics and Computation, vol. 365, Article ID 124718, 2020.

[4] S. Boyd, L. El Ghaoui, E. Feron, and V. Balakrishnan, Linear Matrix Inequalities in Systems and Control Theory, SIAM, Philadelphia, PA, USA, 1994.

[5] T. Hu and Z. Lin, "Composite quadratic Lyapunov functions for constrained control systems," IEEE Transactions on Automatic Control, vol. 48, no. 3, pp. 440-450, 2003.

[6] C. C. Shen and S. M. Zhong, "Improved asymptotic stability criteria for uncertain neutral systems with time-varying discrete delays," International Journal of Information and Mathematical Sciences, vol. 6, p. 1, 2010.

[7] E. Fridman and U. Shaked, "On reachable sets for linear systems with delay and bounded peak inputs," Automatica, vol. 39, no. 11, pp. 2005-2010, 2003.

[8] B. Zhang, J. Lam, and S. Xu, "Relaxed results on reachable set estimation of time-delay systems with bounded peak inputs," International Journal of Robust and Nonlinear Control, vol. 26, no. 9, pp. 1994-2007, 2016.

[9] C. Shen and S. Zhong, "The ellipsoidal bound of reachable sets for linear neutral systems with disturbances," Journal of the Franklin Institute, vol. 348, no. 9, pp. 2570-2585, 2011.

[10] H. Chen and S. M Zhong, "New results on reachable set bounding for linear time delay systems with plytopic uncertainties via novel inequalities," Journal of Inequalities and Applications, vol. 2017, no. 1, p. 277, 2017.

[11] W. Wang, S. Zhong, F. Liu, and J. Cheng, "Reachable set estimation for linear systems with time-varying delay and polytopic uncertainties," Journal of the Franklin Institute, vol. 356, no. 13, pp. 7322-7346, 2019.

[12] P. Balasubramaniam, A. Manivannan, and R. Rakkiyappan, "Exponential stability results for uncertain neutral systems with interval time-varying delays and Markovian jumping parameters," Applied Mathematics and Computation, vol. 216, no. 11, pp. 3396-3407, 2010.

[13] L. Xiong, J. Tian, and X. Liu, "Stability analysis for neutral Markovian jump systems with partially unknown transition probabilities," Journal of the Franklin Institute, vol. 349, no. 6, pp. 2193-2214, 2012.

[14] L. L. Xiong, H. Y. Zhang, and Y. K. Li, "Improved stability and $\mathrm{H}$ performance for neutral systems with uncertain markovian jump," Nonlinear Analysis Hybrid Systems, vol. 19, pp. 13-25, 2016.

[15] W. Chen, B. Zhang, and Q. Ma, "Decay-rate-dependent conditions for exponential stability of stochastic neutral systems with Markovian jumping parameters," Applied Mathematics and Computation, vol. 321, pp. 93-105, 2018.

[16] G. Tartaglione, M. Ariola, and F. Amato, "An observer-based output feedback controller for the finite-time stabilization of markov jump linear systems," IEEE Control Systems Letters, vol. 3, no. 3, pp. 763-768, 2019.

[17] Z. Feng and W. X. Zheng, "On reachable set estimation of delay Markovian jump systems with partially known transition probabilities," Journal of the Franklin Institute, vol. 353, no. 15, pp. 3835-3856, 2016.
[18] Z. Feng, W. X. Zheng, and L. Wu, "Reachable set estimation of T-S fuzzy systems with time-varying delay," IEEE Transactions on Fuzzy Systems, vol. 25, no. 4, pp. 878-891, 2017.

[19] W. J. Lin, Y. He, and M. Wu, "Reachable set estimation for markovian jump neural networks with time-varying delay," Neural Networks, vol. 108, pp. 527-532, 2018.

[20] C. Shen, S. Zhou, and H. Deng, "The No-ellipsoidal bound of reachable sets for neutral markovian jump systems with disturbances," Journal of Applied Mathematics and Physics, vol. 8, no. 5, pp. 799-813, 2020.

[21] A. V. Skorokhod, Asymptotic Methods in the Theory of Stochastic Differential Equation, American Mathematical Society, Providence, RI, USA, 1989.

[22] K. Q. Gu, "An integral inequality in the stability problem of time-delay systems," in Proceedings of the 39th IEEE Conference on Decision and Control, pp. 2805-2810, Sydney, Australia, December 2000.

[23] A. Seuret and F. Gouaisbaut, "Integral inequality for timevarying delay systems," in Proceedings of the European Control Conference (ECC), pp. 3366-3371, Zrich, Switzerland, July 2013.

[24] J. Tian, Z. Ren, and S. Zhong, "A new integral inequality and application to stability of time-delay systems," Applied Mathematics Letters, vol. 101, Article ID 106058, 2020.

[25] P. Park, J. W. Ko, and C. Jeong, "Reciprocally convex approach to stability of systems with time-varying delays," Automatica, vol. 47, no. 1, pp. 235-238, 2011.

[26] J. Tian, L. Xiong, J. Liu, and X. Xie, "Novel delay-dependent robust stability criteria for uncertain neutral systems with time-varying delay," Chaos, Solitons \& Fractals, vol. 40, no. 4, pp. 1858-1866, 2009.

[27] W. Kang, S. Zhong, and K. Shi, "Triple integral approach to reachable set bounding for linear singular systems with timevarying delay," Mathematical Methods in the Applied Sciences, vol. 40, no. 8, pp. 2949-2960, 2017.

[28] J.-H. Kim and F. Jabbari, "Scheduled controllers for buildings under seismic excitation with limited actuator capacity," Journal of Engineering Mechanics, vol. 130, no. 7, pp. 800-808, 2004.

[29] J. Abedor, K. Nagpal, and K. Poolla, "A linear matrix inequality approach to peak-to-peak gain minimization," International Journal of Robust and Nonlinear Control, vol. 6, no. 9-10, pp. 899-927, 1996.

[30] J. K. Hale and S. M. Verduyn Lunel, Introduction to Functional Differential Equation, Springer, New York, NY, USA, 1993. 